TRANSACTIONS OF THE

AMERICAN MATHEMATICAL SOCIETY

Volume 355, Number 11, Pages 4297-4347

S 0002-9947(03)03149-0

Article electronically published on July 2, 2003

\title{
ON CUBIC LACUNARY FOURIER SERIES
}

\author{
JOSEPH L. GERVER
}

\begin{abstract}
For $2<\beta<4$, we analyze the behavior, near the rational points $x=p \pi / q$, of $\sum_{n=1}^{\infty} n^{-\beta} \exp \left(i x n^{3}\right)$, considered as a function of $x$. We expand this series into a constant term, a term on the order of $(x-p \pi / q)^{(\beta-1) / 3}$, a term linear in $x-p \pi / q$, a "chirp" term on the order of $(x-p \pi / q)^{(2 \beta-1) / 4}$, and an error term on the order of $(x-p \pi / q)^{\beta / 2}$. At every such rational point, the left and right derivatives are either both finite (and equal) or both infinite, in contrast with the quadratic series, where the derivative is often finite on one side and infinite on the other. However, in the cubic series, again in contrast with the quadratic case, the chirp term generally has a different set of frequencies and amplitudes on the right and left sides. Finally, we show that almost every irrational point can be closely approximated, in a suitable Diophantine sense, by rational points where the cubic series has an infinite derivative. This implies that when $\beta \leq(\sqrt{97}-1) / 4=2.212 \ldots$, both the real and imaginary parts of the cubic series are differentiable almost nowhere.
\end{abstract}

\section{INTRODUCTION}

The function

$$
F(x)=\sum_{n=1}^{\infty} n^{-2} e^{i x n^{2}}
$$

has been much studied over the past 140 years. It was first investigated by Reimann in an apparent attempt to construct a continuous nowhere differentiable function. Riemann seems to have anticipated much of the later work on this function, but he never published anything on the subject, and although Cristoffel was apparently familiar with Riemann's results [1, they were later forgotten for a century, until Neuenschwander [16] discovered a reference to these results in the diary of Casorati.

Meanwhile, the same results were found independently by Hardy [7] and the author [5], 6]: that $F(x)$ has a derivative of $-\frac{1}{2} i$ at $x=p \pi / q$, where $p$ and $q$ are both odd integers, and is differentiable nowhere else. Interest in $F(x)$ increased about twenty years ago, when Mohr [15, and then Itatsu [11, succeeded in simplifying the proofs in [5], 6], and [7]. Over the past decade, a number of people have simplified the proofs further by use of the wavelet transform, starting with Holschneider and Tchamitchian [10], and continuing with Duistermaat [2], Jaffard [12, and Meyer [13]. (Duistermaat's paper is an excellent self-contained exposition of this method.) As a result of this work, it is now known that $F(x)$ is $C^{1 / 2}$ everywhere, and $C^{3 / 4-\epsilon}$ almost everywhere for each $\epsilon>0$, but $C^{3 / 4}$ nowhere except where it has a derivative, in which case it is $C^{3 / 2-\epsilon}$ for all $\epsilon>0$ but not $C^{3 / 2}$.

Received by the editors October 18, 1999.

2000 Mathematics Subject Classification. Primary 42A55, 26A27.

(C)2003 American Mathematical Society 
An obvious generalization is

$$
F_{\beta}^{\alpha}(x)=\sum_{n=1}^{\infty} n^{-\beta} e^{i x n^{\alpha}} .
$$

We are interested in the case where $\alpha$ is an integer $\geq 2$ and $\beta$ a real number $>1$. It is clear, by termwise differentiation, that $F_{\beta}^{\alpha}(x)$ is everywhere differentiable if $\beta>\alpha+1$. Luther [14] showed that $F_{\beta}^{\alpha}(x)$ is nowhere differentiable if $\beta \leq$ $\alpha-1$. Not much is known when $\alpha-1<\beta \leq \alpha+1$. Hardy showed that $F_{\beta}^{2}(x)$ is almost nowhere differentiable when $\beta<\frac{5}{2}$. Queffelec [17] proved that $F_{\alpha}^{\alpha}(x)$ is differentiable on a dense set (namely at $x=p \pi / q$, where $p$ and $q$ are both odd), and it is straightforward to extend this result to $F_{\beta}^{\alpha}(x)$ when $\beta>\alpha-\frac{1}{2}$. (Luther 14] showed that $F_{\beta}^{\alpha}(x)$ has no derivative at $x=p \pi / q$ when $\beta \leq \alpha-\frac{1}{2}$.) It can also be established easily by standard methods that $F_{\beta}^{2}(x)$ is non-differentiable on a dense set (namely $x=p \pi / q$, where $p$ and $q$ are not both odd) if $\beta<3$.

Thus when $\alpha=2$, we cannot even say what happens almost everywhere if $\frac{5}{2} \leq \beta \leq 3$. When $\alpha>2$, we cannot say what happens almost everywhere if $\alpha-1<\beta \leq \alpha+1$.

One difficulty is that the behavior of $F_{\beta}^{\alpha}(x)$ at the rational point $x=p \pi / q$ depends on the value of the sum

$$
\sum_{k=1}^{2 q} e^{\pi i p k^{\alpha} / q}
$$

If this sum is zero, and $\beta>\alpha-\frac{1}{2}$, then $F_{\beta}^{\alpha}(x)$ has a derivative at $x=p \pi / q$. If the sum is not zero, and $\beta<\alpha+1$, then $F_{\beta}^{\alpha}(x)$ has an infinite left or right derivative there.

It is easy to see that (1.3) must be zero if $p$ and $q$ are both odd. For then $p k^{\alpha}$ and $p(q+k)^{\alpha}$ have opposite parity, whence

$$
p(q+k)^{\alpha} \equiv q+p k^{\alpha} \bmod 2 q
$$

and

$$
e^{\pi i p k^{\alpha} / q}+e^{\pi i p(q+k)^{\alpha} / q}=0
$$

for each $k, 1 \leq k \leq q$.

What happens when $p$ or $q$ is even is completely understood only in the case $\alpha=2$. Then (1.3) is a Gauss sum, equal to $\pm \sqrt{q}$ or $\pm \sqrt{q} i$ when $p$ is even and $q$ odd, and equal to $\sqrt{q}( \pm 1 \pm i)$ when $p$ is odd and $q$ is even. The non-differentiability of $F_{\beta}^{2}(x)$ at the irrational points $x=\xi \pi$ (for $\beta<\frac{5}{2}$ ) follows from the fact that every irrational $\xi$ can be closely approximated (within $c / q^{2}$ ) by an infinite number of rationals $p / q$, with $p$ or $q$ even.

When $\alpha=3$ and $p$ is even, we have

$$
\sum_{k=1}^{2 q} e^{\pi i p k^{3} / q}=2 \Re\left(\sum_{k=1}^{2 q} \chi\left(\frac{k}{q}\right) e^{\pi i p k / q}\right),
$$

where $\chi$ is a cubic character. Suppose $q$ is prime. If $q \equiv 2 \bmod 3$, then $\chi$ is trivial and the sum on the right is zero. This implies that even if $q$ is composite, if it has 
at least one prime factor $\equiv 2 \bmod 3$, and the multiplicity of this prime factor is $\equiv 1 \bmod 3$, then

$$
\sum_{k=1}^{2 q} e^{\pi i p k^{3} / q}=0 .
$$

But the set of integers $q$ satisfying this condition has density 1, leaving a set of density 0 for which $F_{\beta}^{3}(x)$ can have an infinite derivative at $x=p \pi / q$ for some $p$. Such $p / q$ cannot closely approximate every irrational.

Fortunately, by a theorem of Erdös [3], almost all irrationals can be approximated by rationals $p / q$ with $q \in S$, provided the sum over $S$ of $\varphi(q) / q^{2}$ diverges. This holds if $S$ is the set of primes congruent to $1 \bmod 3$. For such primes $q$, we have, for every even $p$ not divisible by $q$,

$$
\left|\sum_{k=1}^{2 q} \chi\left(\frac{k}{q}\right) e^{\pi i p k / q}\right|=2 \sqrt{q} .
$$

The real part of the above sum will be non-zero as long as the sum avoids the imaginary axis. Changing the value of $p$ has the effect of multiplying the sum by a cube root of unity, so if we want $F_{\beta}^{3}(x)$ to have an infinite derivative at $x=p \pi / q$ for all even $p$ (where $q$ does not divide $p$ ), then the sum

$$
\sum_{k=1}^{2 q} \chi\left(\frac{k}{q}\right) e^{2 \pi i k / q}
$$

must avoid the imaginary axis and the two axes which make an angle of $\pi / 3$ with it.

Heath-Brown and Patterson [9] have shown that the argument of (1.9), computed for all primes $q \equiv 1 \bmod 3$, is uniformly distributed on the unit circle. (In fact, we might as well look at (1.9) or its complex conjugate, whichever is in the upper half plane, since (1.9) is only determined up to a complex conjugate unless we specify which cubic character we are using. This doesn't change anything, since we only care about the real part.) So even after we exclude small intervals around the angles $\pi / 6, \pi / 2$, and $5 \pi / 6$, there are enough primes $q$ to satisfy Erdös's condition.

We obtain the following result:

Theorem 1. Both the real and imaginary parts of the function

$$
F_{\beta}(x)=\sum_{n=1}^{\infty} n^{-\beta} e^{i x n^{3}}
$$

have no derivative at $x=\xi \pi$ for almost all irrational numbers $\xi$, provided $\beta \leq$ $(\sqrt{97}-1) / 4=2.212 \ldots$.

This is a bit stronger than Luther's result [14 that $F_{\beta}(x)$ is differentiable nowhere when $\beta \leq 2$. (But note that our result does not imply Luther's, because the price we pay for increasing $\beta$ is to replace "all" with "almost all".) It is likely that the method discussed here can be extended to $\beta \leq \frac{5}{2}$, perhaps even $\beta \leq 3$, but it is hopeless for $\beta>3$. Also, if we try to apply this technique to $F_{\beta}^{\alpha}(x)$, then the upper bound on $\beta$ actually decreases as $\alpha$ increases, so for $\alpha \geq 4$ we cannot improve on Luther (even if the theorem of Heath-Brown and Patterson could be extended to higher powers). 
The proof of Theorem 1 depends on approximating $\xi$ by rationals, and we will make use of two theorems describing the behavior of $F_{\beta}(x)$ near rational multiples of $\pi$. Theorem 2 describes the behavior near $x=0$, and Theorem 3 generalizes this to $x=p \pi / q$. Both theorems express $F_{\beta}(x)$ as the sum of five terms: a constant term, a term proportional to $x^{(\beta-1) / 3}$, a linear term, a "chirp" term of order $x^{(2 \beta-1) / 4}$, and an error term of order $x^{\beta / 2}$. We remark that in the proofs of Theorems 2 and 3 , the case $2<\beta \leq 3$ is handled somewhat differently from the case $3<\beta<4$, and only the former case is needed to prove Theorem 1 .

Theorem 2. Let $x>0$. If $2<\beta<3$, then

$$
\begin{aligned}
\sum_{n=1}^{\infty} \frac{e^{i x n^{3}}}{n^{\beta}}= & \sum_{n=1}^{\infty} \frac{1}{n^{\beta}}+x^{(\beta-1) / 3} \int_{0}^{\infty} \frac{e^{i u^{3}}-1}{u^{\beta}} d u \\
& -2 i x(3-\beta) \int_{0}^{\infty} \frac{\sin u}{u^{\beta-2}} d u \sum_{m=1}^{\infty}(2 \pi m)^{\beta-4} \\
& +(3 x)^{(2 \beta-1) / 4} \int_{-\infty}^{\infty} e^{i u^{2}} d u \sum_{m=1}^{\infty}(2 \pi m)^{-(2 \beta+1) / 4} e^{-\frac{2}{9} \sqrt{3} i(2 \pi m)^{3 / 2} x^{-1 / 2}} \\
& +O\left(x^{\beta / 2}\right)
\end{aligned}
$$

The same is true when $\beta=3$, except that the linear term becomes $-\frac{1}{2} i x$, and when $3<\beta<4$, except that the linear term becomes

$$
i x \lim _{k \rightarrow \infty}\left(\sum_{n=1}^{k} \frac{1}{n^{\beta-3}}-\frac{k^{4-\beta}}{4-\beta}\right) .
$$

Theorem 3. If $x>0$ and $2<\beta \leq 3$, then

$$
F_{\beta}\left(\frac{p \pi}{q}+x\right)-F_{\beta}\left(\frac{p \pi}{q}\right)=H_{1}^{+} x^{(\beta-1) / 3}+H_{2}^{+} i x+H_{3}^{+}(3 x)^{(2 \beta-1) / 4}+H_{4}^{+} x^{\beta / 2}
$$

and

$$
F_{\beta}\left(\frac{p \pi}{q}-x\right)-F_{\beta}\left(\frac{p \pi}{q}\right)=H_{1}^{-} x^{(\beta-1) / 3}+H_{2}^{-} i x+H_{3}^{-}(3 x)^{(2 \beta-1) / 4}+H_{4}^{-} x^{\beta / 2}
$$

where

$$
\begin{aligned}
& H_{1}^{+}=\frac{1}{2 q} \sum_{k=1}^{2 q} e^{\pi i p k^{3} / q} \int_{0}^{\infty} \frac{e^{i u^{3}}-1}{u^{\beta}} d u, \\
& H_{1}^{-}=\frac{1}{2 q} \sum_{k=1}^{2 q} e^{\pi i p k^{3} / q} \int_{0}^{\infty} \frac{e^{-i u^{3}}-1}{u^{\beta}} d u,
\end{aligned}
$$




$$
\begin{aligned}
& H_{2}^{+}=(2 q)^{3-\beta} \sum_{k=1}^{2 q} e^{\pi i p k^{3} / q}\left[\left(\frac{1}{2}-\frac{k}{2 q(4-\beta)}\right)\left(\frac{k}{2 q}\right)^{3-\beta}\right. \\
&\left.+2(3-\beta) \sum_{m=1}^{\infty}(2 \pi m)^{\beta-4} \Re\left(i e^{-\pi i m k / q} \int_{\pi m k / q}^{\infty} \frac{e^{i u}}{u^{\beta-2}} d u\right)\right] \\
& H_{2}^{-}=-(2 q)^{3-\beta} \sum_{k=1}^{2 q} e^{\pi i p k^{3} / q}\left[\left(\frac{1}{2}-\frac{k}{2 q(4-\beta)}\right)\left(\frac{k}{2 q}\right)^{3-\beta}\right.\left.-2(3-\beta) \sum_{m=1}^{\infty}(2 \pi m)^{\beta-4} \Re\left(i e^{-\pi i m k / q} \int_{\pi m k / q}^{\infty} \frac{e^{i u}}{u^{\beta-2}} d u\right)\right] \\
& H_{3}^{+}=(2 q)^{(2 \beta-3) / 4} \int_{-\infty}^{\infty} e^{i u^{2}} d u \\
& \quad \times \sum_{m=1}^{\infty}(2 \pi m)^{-(2 \beta+1) / 4} e^{-\frac{2}{9} \sqrt{3} i(2 \pi m)^{3 / 2}(2 q)^{-3 / 2} x^{-1 / 2}} \sum_{k=1}^{2 q} e^{\pi i\left(p k^{3}+m k\right) / q} \\
& H_{3}^{-}=(2 q)^{(2 \beta-3) / 4} \int_{-\infty}^{\infty} e^{-i u^{2}} d u \\
& \quad \times \sum_{m=1}^{\infty}(2 \pi m)^{-(2 \beta+1) / 4} e^{\frac{2}{9} \sqrt{3} i(2 \pi m)^{3 / 2}(2 q)^{-3 / 2} x^{-1 / 2}} \sum_{k=1}^{2 q} e^{\pi i\left(p k^{3}-m k\right) / q}
\end{aligned}
$$

and both $H_{4}^{+}$and $H_{4}^{-}$are bounded in absolute value by $c_{0} q^{(\beta+2) / 2}$, where $c_{0}$ depends only on $\beta$. If $3<\beta<4$, then the theorem still holds, except that

$$
\begin{aligned}
H_{2}^{+}=\sum_{k=1}^{2 q} e^{\pi i p k^{3} / q}\left(\frac{-k^{4-\beta}}{2 q(4-\beta)}\right. & \\
& \left.\quad+(2 q)^{3-\beta} \lim _{t \rightarrow \infty}\left[\sum_{j=0}^{t-1}\left(j+\frac{k}{2 q}\right)^{3-\beta}-\int_{0}^{t}\left(y+\frac{k}{2 q}\right)^{3-\beta} d y\right]\right)
\end{aligned}
$$

and $H_{2}^{-}=-H_{2}^{+}$.

Although our main goal is to prove Theorem 1, we note that Theorem 3 has some interesting consequences regarding the graphs of the real and imaginary parts of $F_{3}^{3}(x)$, in contrast with $F_{2}^{2}(x)$, near the rational points.

Thus

$$
f_{2}(x)=\sum_{n=1}^{\infty} n^{-2} \sin \left(n^{2} x\right)
$$

has a left derivative of $+\infty$ and a right derivative of $-\frac{1}{2}$ at $x=\pi / 2$ (see Figure 1), and the same thing happens (that is, the derivative is $-\frac{1}{2}$ on one side, and $+\infty$ or $-\infty$ on the other side) at $x=p \pi / q$ whenever $p$ is odd and $q$ is even. This kind of thing never happens with

$$
f_{3}(x)=\sum_{n=1}^{\infty} n^{-3} \sin \left(n^{3} x\right),
$$


where at every rational point, there is a full derivative of $+\infty,-\infty$, or $-\frac{1}{2}$. This is a consequence of the fact that (1.6) is always real.

Now consider the corresponding cosine series.

$$
g_{2}(x)=\sum_{n=1}^{\infty} n^{-2} \cos \left(n^{2} x\right)
$$

behaves much like $f_{2}(x)$. At some rational points, there is a full derivative of $+\infty$ or $-\infty$, at others a full finite derivative, at yet others a left derivative of $+\infty$ and a right derivative of $-\infty$ (or vice versa), and at some a finite left derivative and an infinite right derivative (or vice versa). The main difference between $f_{2}(x)$ and $g_{2}(x)$ is that the finite (left or right) derivative of $f_{2}(x)$ is always $-\frac{1}{2}$, while that of $g_{2}(x)$ is always zero.

With

$$
g_{3}(x)=\sum_{n=1}^{\infty} n^{-3} \cos \left(n^{3} x\right),
$$

on the other hand, if the left derivative is $+\infty$ then the right derivative is $-\infty$ and vice versa. If the left derivative of $g_{3}(x)$ is finite, then it must be equal to the right derivative, but unlike $f_{2}^{\prime}(x), g_{2}^{\prime}(x)$, and $f_{3}^{\prime}(x)$, when $g_{3}^{\prime}(x)$ exists, it is not constrained to always have the same value. For example,

$$
\begin{aligned}
& g_{3}^{\prime}(\pi)=0, \\
& g_{3}^{\prime}(\pi / 3)=-\frac{1}{2} \sqrt{3}, \\
& g_{3}^{\prime}(2 \pi / 3)=-\frac{1}{6} \sqrt{3}, \\
& g_{3}^{\prime}(\pi / 5)=\sin \frac{2 \pi}{5}-\sin \frac{\pi}{5}, \\
& g_{3}^{\prime}(2 \pi / 5)=\frac{1}{5} \sin \frac{\pi}{5}-\frac{3}{5} \sin \frac{2 \pi}{5}
\end{aligned}
$$

(see Figure 2).

The chirp terms are also rather different in the cubic and quadratic cases. The chirp terms of $f_{2}(p \pi / q+x)$ and $g_{2}(p \pi / q+x)$ are always $x^{3 / 2}$ times a periodic function of $x^{-1}$. Indeed, these periodic functions are linear combinations of $f_{2}$ and $g_{2}$ themselves (Figure 3 ). In the cubic case, however, the chirp terms are $x^{5 / 4}$ times quasi-periodic functions of $x^{-1 / 2}$ (Figure 4 ). The quasi-periodicity is a consequnece of the fractional exponent $\frac{3}{2}$ on $2 \pi m$ in $H_{3}^{+}$and $H_{3}^{-}$.

Another difference is that in the quadratic case, the chirp term is always symmetrical. Figure 5 , for example, shows $f_{2}(x)$ near $x=\pi / 3$; the chirp term looks the same to the left and right of $\pi / 3$. But in the cubic case, the two sides of the chirp term can look quite different. Figures 6,7 , and 8 show $f_{3}(x)$ progressively closer to $\pi / 3$. The chirp term is so much larger on the left side, that one must get within $10^{-6}$ of $\pi / 3$ before it is obvious to the eye that $f_{3}(x)$ even has a left derivative there. The asymmetry is caused by the fact that in the sum at the end of $H_{3}^{+}$the exponent is $\pi i\left(p k^{3}+m k\right) / q$, but in $H_{3}^{-}$it is $\pi i\left(p k^{3}-m k\right) / q$. When $p=1$ and $q=3$, the terms are respectively $\exp \left[\pi i\left(k^{3}+m k\right) / 3\right]$ and $\exp \left[\pi i\left(k^{3}-m k\right) / 3\right]$, but since $k^{3} \equiv k \bmod 6$ for all $k$, these are equal to $\exp [\pi i(m+1) k / 3]$ and $\exp [\pi i(m-1) k / 3]$. Now the sum from $k=1$ to 6 of $\exp [\pi i(m+1) k / 3]$ is equal to 6 when $m \equiv 5 \bmod$ 6 , and 0 otherwise, while the sum of $\exp [\pi i(m-1) k / 3]$ is equal to 6 only when 
$m \equiv 1$. Therefore, the first non-zero term of the chirp function to the right of $\pi / 3$ has $5^{3 / 2}$ times the frequency, and $5^{-7 / 4}$ times the amplitude, of the first non-zero term to the left.

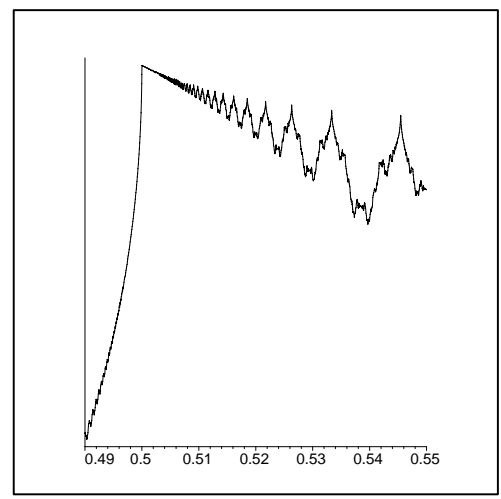

FiguRE 1 (For all figures, the scale on the $x$-axis is in units of $\pi$.)

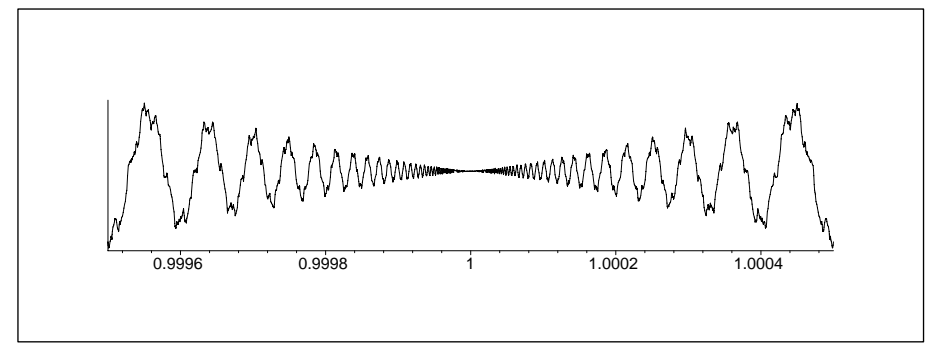

Figure 2(a)

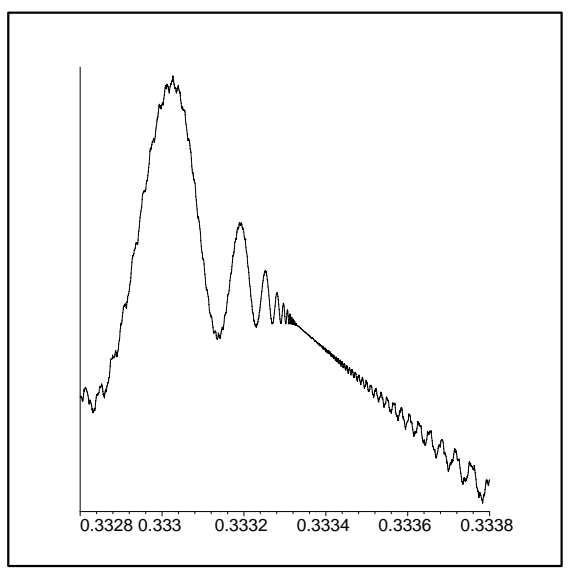

FiguRe 2(b) 


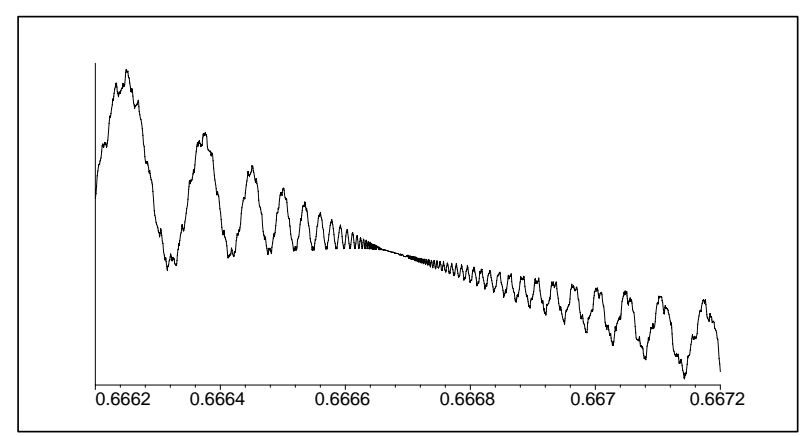

Figure 2(c)

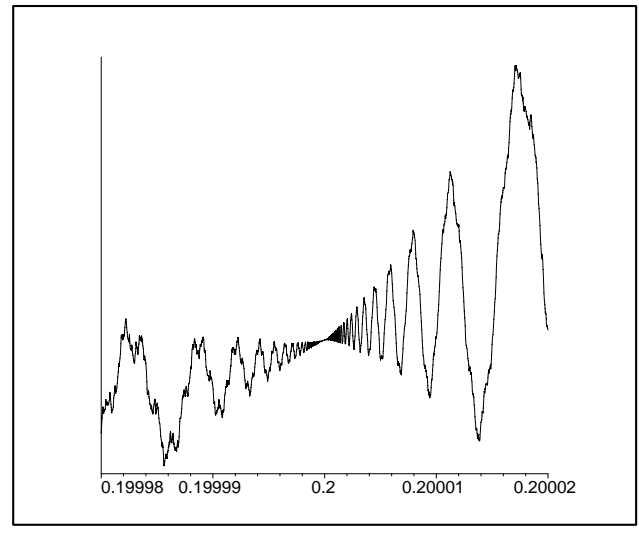

Figure 2(d)

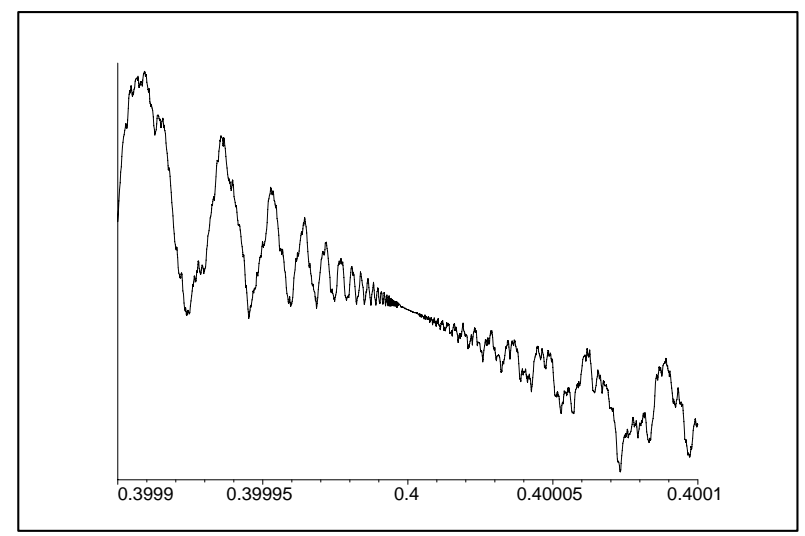

Figure 2(e) 


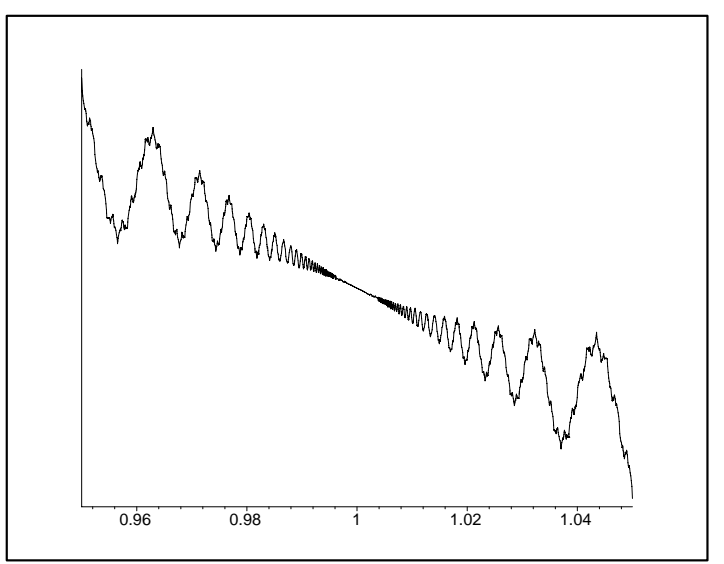

FiguRe 3.

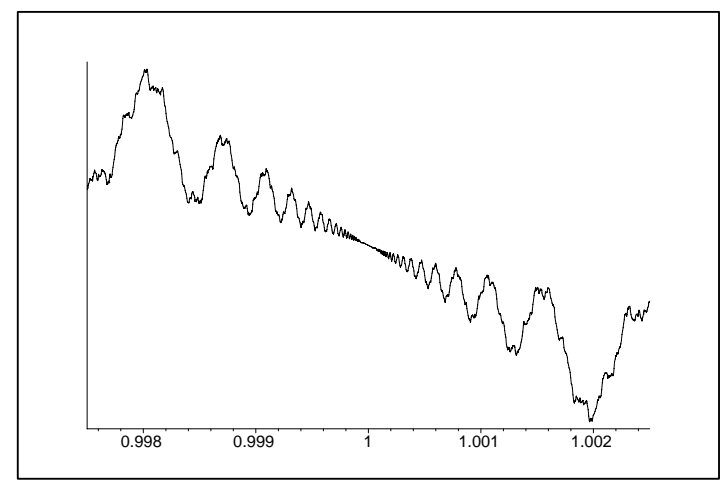

Figure 4.

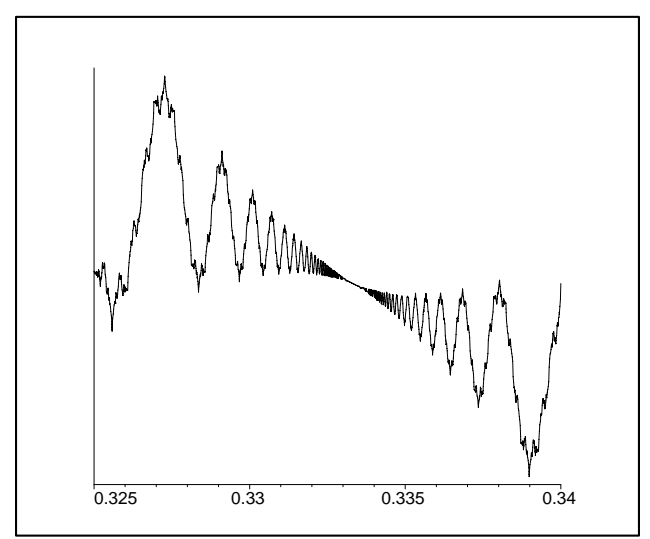

Figure 5. 


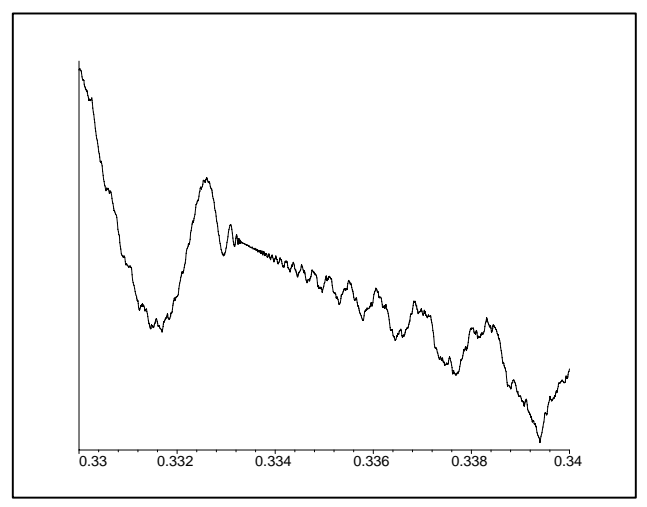

Figure 6.

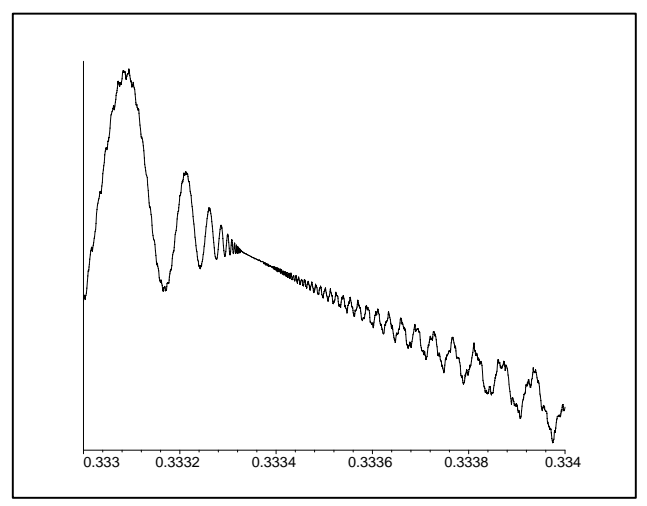

Figure 7.

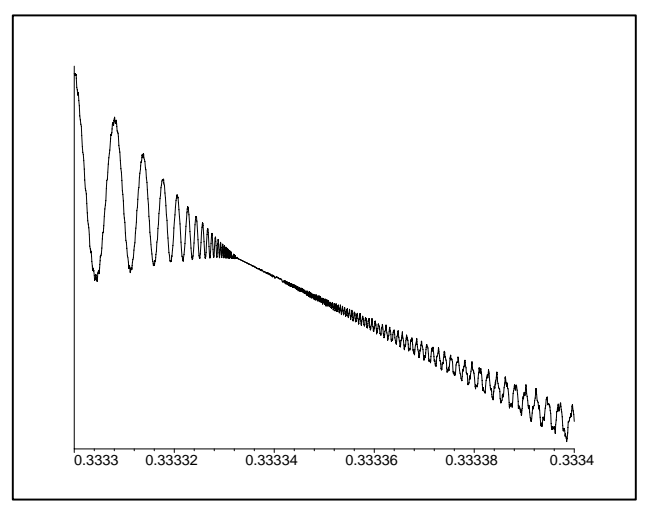

Figure 8. 


\section{BEHAVIOR NEAR ZERO}

Theorem 2, like its quadratic counterpart, is most easily proved using the Poisson summation formula. However, since $\alpha$ is odd in this case, we do not have $\psi(n)=$ $\psi(-n)$, so the sum of $\psi(n)$ over all integers $n$ gives us no direct information about the sum from $n=1$ to $\infty$. Instead, following Hardy and Littlewood [8], we use a one-sided version of the formula.

Lemma 2.1. Let $\psi:[0, \infty) \rightarrow \mathbf{C}$ be continuous and $L^{1}$, and let

$$
\hat{\psi}(t)=2 \int_{0}^{\infty} \psi(y) \cos t y d y .
$$

Then

$$
\psi(0)+2 \sum_{n=1}^{\infty} \psi(n)=\hat{\psi}(0)+2 \sum_{m=1}^{\infty} \hat{\psi}(2 \pi m) .
$$

Proof. Apply the usual (two-sided) Poisson summation formula to $\phi(y)=\psi(|y|)$.

Our main tool for putting bounds on the integrals that we get from Lemma 2.1 is the following lemma.

Lemma 2.2. Let $a$ and $b$ be real numbers, $a<b$, let $r:[a, b] \rightarrow \mathbf{R}^{+}$be $C^{1}$, and let $\theta:[a, b] \rightarrow \mathbf{R}$ be $C^{2}$, with $\theta^{\prime}(u)>0$ for $a \leq u \leq b$. If $r^{\prime}(u) \theta^{\prime}(u)-r(u) \theta^{\prime \prime}(u)<0$ for $a<u<b$, then

$$
\left|\int_{a}^{b} r(u) e^{i \theta(u)} d u\right|<\frac{2 r(a)}{\theta^{\prime}(a)}
$$

If $r^{\prime}(u) \theta^{\prime}(u)-r(u) \theta^{\prime \prime}(u)>0$ for $a<u<b$, then

$$
\left|\int_{a}^{b} r(u) e^{i \theta(u)} d u\right|<\frac{2 r(b)}{\theta^{\prime}(b)} .
$$

Proof. Let

$$
\gamma(t)=\int_{a}^{t} r(u) e^{i \theta(u)} d u
$$

and think of $\gamma(t)$ as a curve in the complex plane, parametrized by $t$. Then the osculating circle to $\gamma(t)$ has radius

$$
\rho(t)=\frac{r(t)}{\theta^{\prime}(t)}
$$

and center

$$
\kappa(t)=\gamma(t)+\frac{i r(t) e^{i \theta(t)}}{\theta^{\prime}(t)}
$$

Now

$$
\rho^{\prime}(t)=\frac{r^{\prime}(t)}{\theta^{\prime}(t)}-\frac{r(t) \theta^{\prime \prime}(t)}{\theta^{\prime}(t)^{2}}=\frac{r^{\prime}(t) \theta^{\prime}(t)-r(t) \theta^{\prime \prime}(t)}{\theta^{\prime}(t)^{2}}
$$

and

$$
\begin{aligned}
\kappa^{\prime}(t) & =\gamma^{\prime}(t)+\frac{i r^{\prime}(t) e^{i \theta(t)}}{\theta^{\prime}(t)}-r(t) e^{i \theta(t)}-\frac{i r(t) \theta^{\prime \prime}(t) e^{i \theta(t)}}{\theta^{\prime}(t)^{2}} \\
& =\frac{i r^{\prime}(t) e^{i \theta(t)}}{\theta^{\prime}(t)}-\frac{i r(t) \theta^{\prime \prime}(t) e^{i \theta(t)}}{\theta^{\prime}(t)^{2}}=i e^{i \theta(t)} \rho^{\prime}(t) .
\end{aligned}
$$


Suppose $r^{\prime}(u) \theta^{\prime}(u)-r(u) \theta^{\prime \prime}(u)<0$ for all $u$ in the interval $a \leq u \leq b$. Then, by (2.2.4), and the fact that $\theta^{\prime}(u)^{2}>0$, we know that $\rho^{\prime}(u)<0$ for all $u$ in this interval. Therefore

$$
\rho(a)>\rho(b)
$$

and

$$
\begin{aligned}
& |\kappa(a)-\kappa(b)|=\left|\int_{a}^{b} \kappa^{\prime}(u) d u\right| \leq \int_{a}^{b}\left|\kappa^{\prime}(u)\right| d u \\
= & \int_{a}^{b}\left|\rho^{\prime}(u)\right| d u=-\int_{a}^{b} \rho^{\prime}(u) d u=\rho(a)-\rho(b) .
\end{aligned}
$$

It follows that the osculating circle at $b$ is entirely inside the osculating circle at $a$. Since $\gamma(b)$ is on the osculating circle at $b, \gamma(b)$ must be inside the osculating circle at $a$, and

$$
|\gamma(b)-\gamma(a)|<2 \rho(a)
$$

The lemma follows. If $r^{\prime}(u) \theta^{\prime}(u)-r(u) \theta^{\prime \prime}(u)>0$, then $\rho^{\prime}(u)>0$, and the same argument applies with $a$ and $b$ reversed.

We are now ready to prove Theorem 2 .

Proof. First we consider the case $2<\beta<3$. We have

$$
\sum_{n=1}^{\infty} \frac{e^{i x n^{3}}}{n^{\beta}}=\sum_{n=1}^{\infty} \frac{1}{n^{\beta}}+\sum_{n=1}^{\infty} \frac{e^{i x n^{3}}-1}{n^{\beta}} .
$$

Fix $x>0$, and let

$$
\psi(y)=\frac{e^{i x y^{3}}-1}{y^{\beta}}
$$

for $y>0$ and

$$
\psi(0)=\lim _{y \rightarrow 0} \frac{e^{i x y^{3}}-1}{y^{\beta}}=0
$$

(recall that $\beta<3$ by hypothesis). Then $\psi$ satisfies the conditions of Lemma 2.1, so

$$
0+2 \sum_{n=1}^{\infty} \frac{e^{i x n^{3}}-1}{n^{\beta}}=2 \int_{0}^{\infty} \frac{e^{i x y^{3}}-1}{y^{\beta}} d y+4 \sum_{m=1}^{\infty} \int_{0}^{\infty} \frac{\left(e^{i x y^{3}}-1\right) \cos (2 \pi m y)}{y^{\beta}} d y
$$

and

$$
\begin{aligned}
\sum_{n=1}^{\infty} \frac{e^{i x n^{3}}-1}{n^{\beta}} & =\int_{0}^{\infty} \frac{e^{i x y^{3}}-1}{y^{\beta}} d y+\sum_{m=1}^{\infty} \int_{0}^{\infty} \frac{\left(e^{i x y^{3}}-1\right) e^{2 \pi i m y}}{y^{\beta}} d y \\
& +\sum_{m=1}^{\infty} \int_{0}^{\infty} \frac{\left(e^{i x y^{3}}-1\right) e^{-2 \pi i m y}}{y^{\beta}} d y .
\end{aligned}
$$

Letting $u=x^{1 / 3} y$, we get

$$
\int_{0}^{\infty} \frac{\left(e^{i x y^{3}}-1\right)}{y^{\beta}} d y=x^{(\beta-1) / 3} \int_{0}^{\infty} \frac{\left(e^{i u^{3}}-1\right)}{u^{\beta}} d u .
$$

Note that the integral on the right converges because $\beta>2$. 
Fix $m$. We will split each of the integrals $\int_{0}^{\infty} y^{-\beta}\left(e^{i x y^{3}}-1\right) e^{2 \pi i m y} d y$ and $\int_{0}^{\infty} y^{-\beta}\left(e^{i x y^{3}}-1\right) e^{-2 \pi i m y} d y$ at $y=\sqrt{2 \pi m / 9 x}$.

Let

$$
g(z)=\int_{0}^{z} \frac{\left(e^{i x y^{3}}-1\right) e^{2 \pi i m y}}{y^{\beta}} d y+\frac{i\left(e^{i x z^{3}}-1\right) e^{2 \pi i m z}}{2 \pi m z^{\beta}} .
$$

Then

$$
g^{\prime}(z)=\frac{-3 x e^{i x z^{3}} e^{2 \pi i m z}}{2 \pi m z^{\beta-2}}-\frac{i \beta\left(e^{i x z^{3}}-1\right) e^{2 \pi i m z}}{2 \pi m z^{\beta+1}} .
$$

Now

$$
\lim _{z \rightarrow 0} g(z)=\lim _{z \rightarrow 0} \frac{i\left(e^{i x z^{3}}-1\right) e^{2 \pi i m z}}{2 \pi m z^{\beta}}=\lim _{z \rightarrow 0} \frac{-x z^{3-\beta}}{2 \pi m}=0
$$

since $\beta<3$, so

$$
\int_{0}^{z} \frac{\left(e^{i x y^{3}}-1\right) e^{2 \pi i m y}}{y^{\beta}} d y=\int_{0}^{z} g^{\prime}(y) d y-\frac{i\left(e^{i x z^{3}}-1\right) e^{2 \pi i m z}}{2 \pi m z^{\beta}} .
$$

We have

$$
g^{\prime}(z)=\frac{-(3-\beta) x e^{2 \pi i m z}}{2 \pi m z^{\beta-2}}-\frac{3 x\left(e^{i x z^{3}}-1\right) e^{2 \pi i m z}}{2 \pi m z^{\beta-2}}-\frac{i \beta\left(e^{i x z^{3}}-1-i x z^{3}\right) e^{2 \pi i m z}}{2 \pi m z^{\beta+1}},
$$

and the substitution $u=2 \pi m y$ gives us

$$
\int_{0}^{\infty} \frac{-(3-\beta) x e^{2 \pi i m y}}{2 \pi m y^{\beta-2}} d y=\frac{-(3-\beta) x}{(2 \pi m)^{4-\beta}} \int_{0}^{\infty} \frac{e^{i u}}{u^{\beta-2}} d u,
$$

So

$$
\begin{aligned}
\int_{0}^{z} g^{\prime}(y) d y & =\frac{-(3-\beta) x}{(2 \pi m)^{4-\beta}} \int_{0}^{\infty} \frac{e^{i u}}{u^{\beta-2}} d u+\int_{z}^{\infty} \frac{(3-\beta) x e^{2 \pi i m y}}{2 \pi m y^{\beta-2}} d y \\
& -\int_{0}^{z} \frac{3 x\left(e^{i x y^{3}}-1\right) e^{2 \pi i m y}}{2 \pi m y^{\beta-2}} d y-\int_{0}^{z} \frac{i \beta\left(e^{i x y^{3}}-1-i x y^{3}\right) e^{2 \pi i m y}}{2 \pi m y^{\beta+1}} d y .
\end{aligned}
$$

Note that Lemma 2.2 guarantees that $\int_{0}^{\infty} u^{-(\beta-2)} e^{i u} d u$ converges for $2<\beta<3$.

Now by Lemma 2.2 , with $r(y)=y^{-(\beta-2)}$ and $\theta(y)=2 \pi m y$, we have

$$
\left|\int_{z}^{\infty} \frac{e^{2 \pi i m y}}{y^{\beta-2}} d y\right|<\frac{1}{\pi m z^{\beta-2}} .
$$

Setting

$$
z=\sqrt{2 \pi m / 9 x}
$$

we have

$$
\int_{z}^{\infty} \frac{(3-\beta) x e^{2 \pi i m y}}{2 \pi m y^{\beta-2}} d y=O\left(x^{\beta / 2} m^{-(\beta+2) / 2}\right) .
$$

Let

$$
\tilde{g}(z)=\int_{0}^{z} \frac{\left(e^{i x y^{3}}-1\right) e^{2 \pi i m y}}{y^{\beta-2}} d y+\frac{i\left(e^{i x z^{3}}-1\right) e^{2 \pi i m z}}{2 \pi m z^{\beta-2}}
$$


and

$$
\hat{g}(z)=\int_{0}^{z} \frac{\left(e^{i x y^{3}}-1-i x y^{3}\right) e^{2 \pi i m y}}{y^{\beta+1}} d y+\frac{i\left(e^{i x z^{3}}-1-i x z^{3}\right) e^{2 \pi i m z}}{2 \pi m z^{\beta+1}} .
$$

Then

$$
\tilde{g}^{\prime}(z)=\frac{-3 x z^{4-\beta} e^{i x z^{3}} e^{2 \pi i m z}}{2 \pi m}-\frac{i(\beta-2)\left(e^{i x z^{3}}-1\right) e^{2 \pi i m z}}{2 \pi m z^{\beta-1}}
$$

and

$$
\hat{g}^{\prime}(z)=\frac{-3 x\left(e^{i x z^{3}}-1\right) e^{2 \pi i m z}}{2 \pi m z^{\beta-1}}-\frac{i(\beta+1)\left(e^{i x z^{3}}-1-i x z^{3}\right) e^{2 \pi i m z}}{2 \pi m z^{\beta+2}} .
$$

Since

$$
\lim _{z \rightarrow 0} \tilde{g}(z)=\lim _{z \rightarrow 0} \frac{-x z^{5-\beta}}{2 \pi m}=0
$$

and

$$
\lim _{z \rightarrow 0} \hat{g}(z)=\lim _{z \rightarrow 0} \frac{-i x^{2} z^{5-\beta}}{4 \pi m}=0
$$

we have

$$
\int_{0}^{z} \frac{\left(e^{i x y^{3}}-1\right) e^{2 \pi i m y}}{y^{\beta-2}} d y=\int_{0}^{z} \tilde{g}^{\prime}(y) d y-\frac{i\left(e^{i x z^{3}}-1\right) e^{2 \pi i m z}}{2 \pi m z^{\beta-2}}
$$

and

$$
\int_{0}^{z} \frac{\left(e^{i x y^{3}}-1-i x y^{3}\right) e^{2 \pi i m y}}{y^{\beta+1}} d y=\int_{0}^{z} \hat{g}^{\prime}(y) d y-\frac{i\left(e^{i x z^{3}}-1-i x z^{3}\right) e^{2 \pi i m z}}{2 \pi m z^{\beta+1}}
$$

Now

$$
\int_{0}^{z} \tilde{g}^{\prime}(y) d y=\frac{-3 x}{2 \pi m} \int_{0}^{z} y^{4-\beta} e^{i\left(x y^{3}+2 \pi m y\right)} d y-\frac{i(\beta-2)}{2 \pi m} \int_{0}^{z} \frac{\left(e^{i x y^{3}}-1\right) e^{2 \pi i m y}}{y^{\beta-1}} d y .
$$

Applying Lemma 2.2 to the first integral on the right of (2.25), we get $r(y)=y^{4-\beta}$ and $\theta(y)=x y^{3}+2 \pi m y$, so

$$
r^{\prime}(y) \theta^{\prime}(y)-r(y) \theta^{\prime \prime}(y)=-3(\beta-2) x y^{5-\beta}+2 \pi(4-\beta) m y^{3-\beta} .
$$

Thus $r^{\prime}(y) \theta^{\prime}(y)-r(y) \theta^{\prime \prime}(y)>0$ if

$$
0<y<\sqrt{\frac{2 \pi m(4-\beta)}{3 x(\beta-2)}} .
$$

But $2<\beta<3$, so $(4-\beta) /(\beta-2)>1$, and $r^{\prime}(y) \theta^{\prime}(y)-r(y) \theta^{\prime \prime}(y)>0$ for all $y$ between 0 and $\sqrt{2 \pi m / 9 x}$. It follows from Lemma 2.2 that when (2.15) holds,

$$
\left|\int_{0}^{z} y^{4-\beta} e^{i\left(x y^{3}+2 \pi m y\right)} d y\right|<\frac{2 r(z)}{\theta^{\prime}(z)}=\frac{2 z^{4-\beta}}{3 x z^{2}+2 \pi m}=O\left(x^{(\beta-4) / 2} m^{(2-\beta) / 2}\right) .
$$

Therefore

$$
\frac{-3 x}{2 \pi m} \int_{0}^{z} y^{4-\beta} e^{i\left(x y^{3}+2 \pi m y\right)} d y=O\left(x^{(\beta-2) / 2} m^{-\beta / 2}\right) .
$$


We compute the second integral on the right-hand side of (2.25) in two pieces, from $y=0$ to $x^{-1 / 3}$ and from $y=x^{-1 / 3}$ to $z$. We have

$$
e^{i x y^{3}}-1=\sum_{k=1}^{\infty} \frac{\left(i x y^{3}\right)^{k}}{k !}
$$

So

$$
\int_{0}^{x^{-1 / 3}} \frac{\left(e^{i x y^{3}}-1\right) e^{2 \pi i m y}}{y^{\beta-1}} d y=\sum_{k=1}^{\infty} \frac{(i x)^{k}}{k !} \int_{0}^{x^{-1 / 3}} y^{3 k+1-\beta} e^{2 \pi i m y} d y .
$$

By Lemma 2.2,

$$
\left|\int_{0}^{x^{-1 / 3}} y^{3 k+1-\beta} e^{2 \pi i m y} d y\right|<\frac{2 x^{(\beta-1) / 3-k}}{2 \pi m}
$$

SO

$$
\begin{gathered}
\left|\int_{0}^{x^{-1 / 3}} \frac{\left(e^{i x y^{3}}-1\right) e^{2 \pi i m y}}{y^{\beta-1}} d y\right| \leq \sum_{k=1}^{\infty} \frac{x^{k}}{k !}\left|\int_{0}^{x^{-1 / 3}} y^{3 k+1-\beta} e^{2 \pi i m y} d y\right| \\
<\sum_{k=1}^{\infty} \frac{x^{(\beta-1) / 3}}{\pi m k !}=\frac{(e-1) x^{(\beta-1) / 3}}{\pi m} .
\end{gathered}
$$

For $x^{-1 / 3}<y<z$, where (2.15) continues to hold, we have

$$
\int_{x^{-1 / 3}}^{z} \frac{\left(e^{i x y^{3}}-1\right) e^{2 \pi i m y}}{y^{\beta-1}} d y=\int_{x^{-1 / 3}}^{z} \frac{e^{i\left(x y^{3}+2 \pi m y\right)}}{y^{\beta-1}} d y-\int_{x^{-1 / 3}}^{z} \frac{e^{2 \pi i m y}}{y^{\beta-1}} d y .
$$

Applying Lemma 2.2 to each of the integrals on the right-hand side of (2.34), we get

$$
\left|\int_{x^{-1 / 3}}^{z} \frac{\left(e^{i x y^{3}}-1\right) e^{2 \pi i m y}}{y^{\beta-1}} d y\right| \leq \frac{2 x^{(\beta-1) / 3}}{3 x^{1 / 3}+2 \pi m}+\frac{2 x^{(\beta-1) / 3}}{2 \pi m} .
$$

Combining (2.33) and (2.35), we find that

$$
\int_{0}^{z} \frac{\left(e^{i x y^{3}}-1\right) e^{2 \pi i m y}}{y^{\beta-1}} d y=O\left(x^{(\beta-1) / 3} m^{-1}\right)
$$

and

$$
\frac{-i(\beta-2)}{2 \pi m} \int_{0}^{z} \frac{\left(e^{i x y^{3}}-1\right) e^{2 \pi i m y}}{y^{\beta-1}} d y=O\left(x^{(\beta-1) / 3} m^{-2}\right) .
$$

Since $2<\beta<3$, we have $\beta / 2<2$ and $(\beta-2) / 2<(\beta-1) / 3$. Therefore, by $(2.25)$, $(2.29)$, and $(2.37)$, we have

$$
\int_{0}^{z} \tilde{g}^{\prime}(y) d y=O\left(x^{(\beta-2) / 2} m^{-\beta / 2}\right)
$$

and, with (2.23),

$$
\begin{aligned}
\int_{0}^{z} \frac{\left(e^{i x y^{3}}-1\right) e^{2 \pi i m y}}{y^{\beta-2}} d y & =O\left(x^{(\beta-2) / 2} m^{-\beta / 2}\right)+O\left(z^{-(\beta-2)} m^{-1}\right) \\
& =O\left(x^{(\beta-2) / 2} m^{-\beta / 2}\right)
\end{aligned}
$$


Next, we have

$$
\begin{aligned}
\int_{0}^{z} \hat{g}^{\prime}(y) d y= & \frac{-3 x}{2 \pi m} \int_{0}^{z} \frac{\left(e^{i x y^{3}}-1\right) e^{2 \pi i m y}}{y^{\beta-1}} d y \\
& \quad-\frac{i(\beta+1)}{2 \pi m} \int_{0}^{z} \frac{\left(e^{i x y^{3}}-1-i x y^{3}\right) e^{2 \pi i m y}}{y^{\beta+2}} d y .
\end{aligned}
$$

The first integral on the right of (2.40) is the same as (2.36). To put a bound on the second integral, we note that

$$
e^{i x y^{3}}-1-i x y^{3}=\sum_{k=2}^{\infty} \frac{\left(i x y^{3}\right)^{k}}{k !}
$$

$\mathrm{SO}$

$$
\int_{0}^{x^{-1 / 3}} \frac{\left(e^{i x y^{3}}-1-i x y^{3}\right) e^{2 \pi i m y}}{y^{\beta+2}} d y=\sum_{k=2}^{\infty} \frac{(i x)^{k}}{k !} \int_{0}^{x^{-1 / 3}} y^{3 k-2-\beta} e^{2 \pi i m y} d y .
$$

By Lemma 2.2, for each $k \geq 2$,

$$
\left|\int_{0}^{x^{-1 / 3}} y^{3 k-2-\beta} e^{2 \pi i m y} d y\right|<\frac{2 x^{(\beta+2) / 3-k}}{2 \pi m}
$$

so

$$
\left|\int_{0}^{x^{-1 / 3}} \frac{\left(e^{i x y^{3}}-1-i x y^{3}\right) e^{2 \pi i m y}}{y^{\beta+2}} d y\right|<\sum_{k=2}^{\infty} \frac{x^{(\beta+2) / 3}}{\pi m k !}=\frac{(e-2) x^{(\beta+2) / 3}}{\pi m} .
$$

For $x^{-1 / 3}<y<z=\sqrt{2 \pi m / 9 x}$, we have

$$
\begin{aligned}
\int_{x^{-1 / 3}}^{z} & \frac{\left(e^{i x y^{3}}-1-i x y^{3}\right) e^{2 \pi i m y}}{y^{\beta+2}} d y \\
& =\int_{x^{-1 / 3}}^{z} \frac{e^{i\left(x y^{3}+2 \pi m y\right)}}{y^{\beta+2}} d y-\int_{x^{-1 / 3}}^{z} \frac{e^{2 \pi i m y}}{y^{\beta+2}} d y-i x \int_{x^{-1 / 3}}^{z} \frac{e^{2 \pi i m y}}{y^{\beta-1}} d y .
\end{aligned}
$$

Applying Lemma 2.2 to each integral on the right of $(2.45)$, we get $O\left(x^{(\beta+2) / 3} m^{-1}\right)$ for each term. Combining this bound with (2.44), we obtain

$$
\int_{0}^{z} \frac{\left(e^{i x y^{3}}-1-i x y^{3}\right) e^{2 \pi i m y}}{y^{\beta+2}} d y=O\left(x^{(\beta+2) / 3} m^{-1}\right) .
$$

Finally, combining (2.40), (2.36), and (2.46), we get

$$
\int_{0}^{z} \hat{g}^{\prime}(y) d y=O\left(x^{(\beta+2) / 3} m^{-2}\right)=O\left(x^{\beta / 2} m^{-2}\right)
$$

and, by (2.24),

$$
\begin{gathered}
\int_{0}^{z} \frac{\left(e^{i x y^{3}}-1-i x y^{3}\right) e^{2 \pi i m y}}{y^{\beta+1}} d y \\
=O\left(x^{\beta / 2} m^{-2}\right)+O\left(z^{-(\beta+1)} m^{-1}\right)+O\left(x z^{-(\beta-2)} m^{-1}\right)=O\left(x^{\beta / 2} m^{-\beta / 2}\right) .
\end{gathered}
$$

It then follows from (2.13), (2.16), (2.39), and (2.48) that

$$
\int_{0}^{z} g^{\prime}(y) d y=\frac{-(3-\beta) x}{(2 \pi m)^{4-\beta}} \int_{0}^{\infty} \frac{e^{i u}}{u^{\beta-2}} d u+O\left(x^{\beta / 2} m^{-(\beta+2) / 2}\right),
$$


and, with (2.10), we have

$$
\int_{0}^{z} \frac{\left(e^{i x y^{3}}-1\right) e^{2 \pi i m y}}{y^{\beta}} d y=\frac{-(3-\beta) x}{(2 \pi m)^{4-\beta}} \int_{0}^{\infty} \frac{e^{i u}}{u^{\beta-2}} d u+O\left(x^{\beta / 2} m^{-(\beta+2) / 2}\right) .
$$

The integral of $y^{-\beta}\left(e^{i x y^{3}}-1\right) e^{-2 \pi i m y}$ is handled in a similar fashion, except that Lemma 2.2, instead of being applied directly to integrals of the form

$$
\int_{a}^{b} y^{\gamma} e^{-2 \pi i m y} d y
$$

and

$$
\int_{a}^{b} y^{\gamma} e^{i\left(x y^{3}-2 \pi m y\right)} d y
$$

is applied to the complex conjugates of these integrals (which of course have the same absolute value), so that $\theta^{\prime}(y)>0$. This makes no difference for integrals of the form (2.51), because $\theta(y)$ is still $2 \pi m y$. But for the complex conjugate of (2.52), $\theta(y)=2 \pi m y-x y^{3}$, and the conditions $\theta^{\prime}(y)>0, r^{\prime}(u) \theta^{\prime}(u)-r(u) \theta^{\prime \prime}(u)<0$, and $r^{\prime}(u) \theta^{\prime}(u)-r(u) \theta^{\prime \prime}(u)>0$ will not be satisfied for the same ranges of $y$ as before. Indeed, $\theta^{\prime}(y)=2 \pi m-3 x y^{2}$, so $\theta^{\prime}(y)>0$ only for $y<\sqrt{2 \pi m / 3 x}$; but this is automatically satisfied when $y<\sqrt{2 \pi m / 9 x}$. When $\gamma$ is positive, an in (2.28), we have $r^{\prime}(u) \theta^{\prime}(u)-r(u) \theta^{\prime \prime}(u)>0$ for all $y$, because $\theta^{\prime \prime}(y)<0$. However, when $\gamma$ is negative, as in the first integrals on the right-hand sides of (2.34) and (2.45), we will have additional restrictions on $y$. In particular, if $r(y)=y^{\gamma}$ and $\theta(y)=2 \pi m y-x y^{3}$, then

$$
r^{\prime}(y) \theta^{\prime}(y)=\gamma y^{\gamma-1}\left(2 \pi m-3 x y^{2}\right)=2 \pi \gamma m y^{\gamma-1}-3 \gamma x y^{\gamma+1}
$$

and

$$
r(y) \theta^{\prime \prime}(y)=-6 x y^{\gamma+1}
$$

so $r^{\prime}(u) \theta^{\prime}(u)-r(u) \theta^{\prime \prime}(u)<0$ if and only if $y^{2}(\gamma-2)>2 \pi m \gamma / 3 x$. In (2.34), $\gamma=-(\beta-1)$, so the first part of Lemma 2.2 holds as long as

$$
y<\sqrt{2 \pi m(\beta-1) / 3 x(\beta+1)} .
$$

In $(2.45), \gamma=-(\beta+2)$, so the bound is

$$
y<\sqrt{2 \pi m(\beta+2) / 3 x(\beta+4)} .
$$

In both cases, we can apply Lemma 2.2 whenever $y<\sqrt{2 \pi m / 9 x}$, since $\beta>2$. It follows that when (2.15) holds,

$$
\int_{0}^{z} \frac{\left(e^{i x y^{3}}-1\right) e^{-2 \pi i m y}}{y^{\beta}} d y=\frac{(3-\beta) x}{(2 \pi m)^{4-\beta}} \int_{0}^{\infty} \frac{e^{-i u}}{u^{\beta-2}} d u+O\left(x^{\beta / 2} m^{-(\beta+2) / 2}\right) .
$$

Note that the second term on the right in (2.7) must be negative in this case, so that the first term on the right, which is negative in (2.50), becomes positive in (2.53).

To complete the proof, we must estimate

$$
\int_{z}^{\infty} \frac{\left(e^{i x y^{3}}-1\right) e^{2 \pi i m y}}{y^{\beta}} d y
$$

and

$$
\int_{z}^{\infty} \frac{\left(e^{i x y^{3}}-1\right) e^{-2 \pi i m y}}{y^{\beta}} d y
$$


By Lemma 2.2, we have

$$
\left|\int_{z}^{\infty} \frac{e^{2 \pi i m y}}{y^{\beta}} d y\right|=\left|\int_{z}^{\infty} \frac{e^{-2 \pi i m y}}{y^{\beta}} d y\right|<\frac{2}{2 \pi m z^{\beta}}=O\left(x^{\beta / 2} m^{-(\beta+2) / 2}\right)
$$

and

$$
\int_{z}^{\infty} \frac{e^{i\left(x y^{3}+2 \pi m y\right)}}{y^{\beta}} d y=O\left(x^{\beta / 2} m^{-(\beta+2) / 2}\right) .
$$

This leaves

$$
\int_{z}^{\infty} \frac{e^{i\left(x y^{3}-2 \pi m y\right)}}{y^{\beta}} d y
$$

but this integral behaves quite differently from (2.57), because $\theta^{\prime}(y)=3 x y^{2}-$ $2 \pi m$, which is zero at $y=\sqrt{2 \pi m / 3 x}$. Indeed, it is (2.58), in the vicinity of $y=\sqrt{2 \pi m / 3 x}$, which is responsible for the "chirp" term proportional to $x^{(2 \beta-1) / 4}$ in Theorem 2 .

We therefore evaluate $(2.58)$ by expanding around $y=\sqrt{2 \pi m / 3 x}$. In particular, let

$$
y=(6 \pi m x)^{-1 / 4} u+\sqrt{2 \pi m / 3 x},
$$

let

$$
c=(6 \pi m)^{-3 / 4} x^{1 / 4},
$$

and let

$$
w=\left(\frac{1}{3}-\frac{1}{9} \sqrt{3}\right) c^{-1},
$$

so that

$$
\begin{gathered}
\int_{z}^{\infty} \frac{e^{i\left(x y^{3}-2 \pi m y\right)}}{y^{\beta}} d y \\
=(2 \pi m)^{-(2 \beta+1) / 4}(3 x)^{(2 \beta-1) / 4} e^{-\frac{2}{9} \sqrt{3} i(2 \pi m)^{3 / 2} x^{-1 / 2}} \int_{-w}^{\infty} \frac{e^{i\left(u^{2}+c u^{3}\right)}}{(1+3 c u)^{\beta}} d u .
\end{gathered}
$$

We want to show that

$$
\int_{-w}^{\infty} \frac{e^{i\left(u^{2}+c u^{3}\right)}}{(1+3 c u)^{\beta}} d u=\int_{-\infty}^{\infty} e^{i u^{2}} d u+O(c) .
$$

By Lemma 2.2,

$$
\left|\int_{w}^{\infty} \frac{e^{i\left(u^{2}+c u^{3}\right)}}{(1+3 c u)^{\beta}} d u\right|<\frac{2\left(2-\frac{1}{3} \sqrt{3}\right)^{-\beta}}{2\left(\frac{1}{3}-\frac{1}{9} \sqrt{3}\right) c^{-1}+3\left(\frac{1}{3}-\frac{1}{9} \sqrt{3}\right)^{2} c^{-1}}=O(c),
$$

SO

$$
\int_{-w}^{\infty} \frac{e^{i\left(u^{2}+c u^{3}\right)}}{(1+3 c u)^{\beta}} d u=\int_{-w}^{w} \frac{e^{i\left(u^{2}+c u^{3}\right)}}{(1+3 c u)^{\beta}} d u+O(c) .
$$

We also have

$$
\int_{-\infty}^{\infty} e^{i u^{2}} d u=\int_{-w}^{w} e^{i u^{2}} d u+O(c)
$$

Now

$$
\int_{-w}^{w} \frac{e^{i\left(u^{2}+c u^{3}\right)}}{(1+3 c u)^{\beta}} d u=\int_{0}^{w} \frac{e^{i\left(u^{2}+c u^{3}\right)}}{(1+3 c u)^{\beta}} d u+\int_{0}^{w} \frac{e^{i\left(u^{2}-c u^{3}\right)}}{(1-3 c u)^{\beta}} d u
$$


and

$$
\int_{-w}^{w} e^{i u^{2}} d u=2 \int_{0}^{w} e^{i u^{2}} d u
$$

so it suffices to show that

$$
\int_{0}^{w} \frac{e^{i\left(u^{2}+c u^{3}\right)}}{(1+3 c u)^{\beta}} d u+\int_{0}^{w} \frac{e^{i\left(u^{2}-c u^{3}\right)}}{(1-3 c u)^{\beta}} d u-2 \int_{0}^{w} e^{i u^{2}} d u=O(c) .
$$

We will evaluate these integrals in two pieces, from $u=0$ to 1 and from $u=1$ to $w$. We start with the second interval.

Let

$$
\begin{gathered}
g_{0}(z)=\int_{0}^{z} e^{i u^{2}} d u+\frac{i e^{i z^{2}}}{2 z}, \\
g_{1}(z)=\int_{0}^{z} \frac{e^{i\left(u^{2}+c u^{3}\right)}}{(1+3 c u)^{\beta}} d u+\frac{i e^{i\left(z^{2}+c z^{3}\right)}}{z(2+3 c z)(1+3 c z)^{\beta}},
\end{gathered}
$$

and

$$
g_{2}(z)=\int_{0}^{z} \frac{e^{i\left(u^{2}-c u^{3}\right)}}{(1-3 c u)^{\beta}} d u+\frac{i e^{i\left(z^{2}-c z^{3}\right)}}{z(2-3 c z)(1-3 c z)^{\beta}}
$$

Then

$$
g_{0}^{\prime}(z)=\frac{-i e^{i z^{2}}}{2 z^{2}}
$$

and

$g_{1}^{\prime}(z)=\frac{-3 i \beta c e^{i\left(z^{2}+c z^{3}\right)}}{z(2+3 c z)(1+3 c z)^{\beta+1}}-\frac{i e^{i\left(z^{2}+c z^{3}\right)}}{z^{2}(2+3 c z)(1+3 c z)^{\beta}}-\frac{3 i c e^{i\left(z^{2}+c z^{3}\right)}}{z(2+3 c z)^{2}(1+3 c z)^{\beta}}$,

SO

$$
\begin{aligned}
& g_{1}^{\prime}(z)-g_{0}^{\prime}(z)=\frac{-3 i \beta c e^{i\left(z^{2}+c z^{3}\right)}}{z(2+3 c z)(1+3 c z)^{\beta+1}}-\frac{3 i c e^{i\left(z^{2}+c z^{3}\right)}}{z(2+3 c z)^{2}(1+3 c z)^{\beta}} \\
& -\frac{i e^{i z^{2}}\left(e^{i c z^{3}}-1\right)}{2 z^{2}}+\frac{i e^{i\left(z^{2}+c z^{3}\right)}\left[(2+3 c z)(1+3 c z)^{\beta}-2\right]}{2 z^{2}(2+3 c z)(1+3 c z)^{\beta}}
\end{aligned}
$$

Likewise

$$
\begin{aligned}
& g_{2}^{\prime}(z)-g_{0}^{\prime}(z)=\frac{3 i \beta c e^{i\left(z^{2}-c z^{3}\right)}}{z(2-3 c z)(1-3 c z)^{\beta+1}}+\frac{3 i c e^{i\left(z^{2}-c z^{3}\right)}}{z(2-3 c z)^{2}(1-3 c z)^{\beta}} \\
& -\frac{i e^{i z^{2}}\left(e^{-i c z^{3}}-1\right)}{2 z^{2}}+\frac{i e^{i\left(z^{2}-c z^{3}\right)}\left[(2-3 c z)(1-3 c z)^{\beta}-2\right]}{2 z^{2}(2-3 c z)(1-3 c z)^{\beta}}
\end{aligned}
$$

and

$$
\begin{gathered}
g_{1}^{\prime}(z)+g_{2}^{\prime}(z)-2 g_{0}^{\prime}(z)=\frac{-3 i \beta c e^{i\left(z^{2}+c z^{3}\right)}}{z(2+3 c z)(1+3 c z)^{\beta+1}}+\frac{3 i \beta c e^{i\left(z^{2}-c z^{3}\right)}}{z(2-3 c z)(1-3 c z)^{\beta+1}} \\
-\frac{3 i c e^{i\left(z^{2}+c z^{3}\right)}}{z(2+3 c z)^{2}(1+3 c z)^{\beta}}+\frac{3 i c e^{i\left(z^{2}-c z^{3}\right)}}{z(2-3 c z)^{2}(1-3 c z)^{\beta}}+\frac{i e^{i z^{2}}\left[1-\cos \left(c z^{3}\right)\right]}{z^{2}} \\
\frac{i e^{i\left(z^{2}+c z^{3}\right)}\left[(2+3 c z)(1+3 c z)^{\beta}-2\right]}{2 z^{2}(2+3 c z)(1+3 c z)^{\beta}}+\frac{i e^{i\left(z^{2}-c z^{3}\right)}\left[(2-3 c z)(1-3 c z)^{\beta}-2\right]}{2 z^{2}(2-3 c z)(1-3 c z)^{\beta}} .
\end{gathered}
$$


Now

$$
\begin{aligned}
& \int_{1}^{w} \frac{e^{i\left(u^{2}+c u^{3}\right)}}{(1+3 c u)^{\beta}} d u+\int_{1}^{w} \frac{e^{i\left(u^{2}-c u^{3}\right)}}{(1-3 c u)^{\beta}} d u-2 \int_{1}^{w} e^{i u^{2}} d u \\
& =\int_{1}^{w}\left[g_{1}^{\prime}(u)+g_{2}^{\prime}(u)-2 g_{0}^{\prime}(u)\right] d u-\frac{i e^{i\left(w^{2}+c w^{3}\right)}}{w(2+3 c w)(1+3 c w)^{\beta}} \\
& -\frac{i e^{i\left(w^{2}-c w^{3}\right)}}{w(2-3 c w)(1-3 c w)^{\beta}}+\frac{i e^{i w^{2}}}{w}+\frac{i e^{i(1+c)}}{(2+3 c)(1+3 c)^{\beta}}+\frac{i e^{i(1-c)}}{(2-3 c)(1-3 c)^{\beta}}-i e^{i} .
\end{aligned}
$$

We have

$$
\frac{-i e^{i\left(w^{2}+c w^{3}\right)}}{w(2+3 c w)(1+3 c w)^{\beta}}-\frac{i e^{i\left(w^{2}-c w^{3}\right)}}{w(2-3 c w)(1-3 c w)^{\beta}}+\frac{i e^{i w^{2}}}{w}=O\left(w^{-1}\right)=O(c)
$$

and

$$
\begin{gathered}
\frac{i e^{i(1+c)}}{(2+3 c)(1+3 c)^{\beta}}+\frac{i e^{i(1-c)}}{(2-3 c)(1-3 c)^{\beta}}-i e^{i} \\
=\frac{i e^{i(1+c)}\left[2-(2+3 c)(1+3 c)^{\beta}\right]}{2(2+3 c)(1+3 c)^{\beta}}+\frac{i e^{i(1-c)}\left[2-(2-3 c)(1-3 c)^{\beta}\right]}{2(2-3 c)(1-3 c)^{\beta}} \\
+i e^{i}(\cos c-1)=O(c) .
\end{gathered}
$$

Therefore, in order to prove that the left-hand side of $(2.78)$ is $O(c)$, it suffices to show that

$$
\int_{1}^{w}\left[g_{1}^{\prime}(u)+g_{2}^{\prime}(u)-2 g_{0}^{\prime}(u)\right] d u=O(c)
$$

We will do this by applying Lemma 2.2 to each term on the right of $(2.77)$.

For the first term, ignoring the constant factor of $-3 i \beta c$, we have

$$
r(u)=u^{-1}(2+3 c u)^{-1}(1+3 c u)^{-\beta-1} \quad \text { and } \quad \theta(u)=u^{2}+c u^{3},
$$

SO

$$
r^{\prime}(u) \theta^{\prime}(u)-r(u) \theta^{\prime \prime}(u)=\frac{-9(\beta+5) c^{2} u^{2}-6(\beta+5) c u-4}{u(2+3 c u)(1+3 c u)^{\beta+2}},
$$

which is negative for $c u>0$. It follows from Lemma 2.2 that

$$
\left|\int_{1}^{w} \frac{e^{i\left(u^{2}+c u^{3}\right)}}{u(2+3 c u)(1+3 c u)^{\beta+1}} d u\right|<\frac{2 r(1)}{\theta^{\prime}(1)}=\frac{2}{(2+3 c)^{2}(1+3 c)^{\beta+1}}=O(1)
$$

SO

$$
\int_{1}^{w} \frac{-3 i \beta c e^{i\left(u^{2}+c u^{3}\right)}}{u(2+3 c u)(1+3 c u)^{\beta+1}} d u=O(c) .
$$

For the second term, $r(u)=u^{-1}(2-3 c u)^{-1}(1-3 c u)^{-\beta-1}$ and $\theta(u)=u^{2}-c u^{3}$, SO

$$
r^{\prime}(u) \theta^{\prime}(u)-r(u) \theta^{\prime \prime}(u)=\frac{-9(\beta+5) c^{2} u^{2}+6(\beta+5) c u-4}{u(2-3 c u)(1-3 c u)^{\beta+2}} .
$$

The denominator is positive for $0<c u<\frac{1}{3}$, and the numerator is negative for $c u<\frac{1}{3}-\frac{1}{3} \sqrt{1-4 /(\beta+5)}$ and positive for $\frac{1}{3}-\frac{1}{3} \sqrt{1-4 /(\beta+5)}<c u<\frac{1}{3}+$ 
$\frac{1}{3} \sqrt{1-4 /(\beta+5)}$. It follows from Lemma 2.2 that

$$
\left|\int_{1}^{\frac{1}{3}(1-\sqrt{1-4 /(\beta+5)}) c^{-1}} \frac{e^{i\left(u^{2}-c u^{3}\right)}}{u(2-3 c u)(1-3 c u)^{\beta+1}} d u\right|<\frac{2 r(1)}{\theta^{\prime}(1)}=O(1)
$$

and

$$
\left|\int_{\frac{1}{3}(1-\sqrt{1-4 /(\beta+5)}) c^{-1}}^{w} \frac{e^{i\left(u^{2}-c u^{3}\right)}}{u(2-3 c u)(1-3 c u)^{\beta+1}} d u\right|<\frac{2 r(w)}{\theta^{\prime}(w)}=O\left(c^{2}\right) .
$$

Therefore

$$
\int_{1}^{w} \frac{-3 i \beta c e^{i\left(u^{2}-c u^{3}\right)}}{u(2-3 c u)(1-3 c u)^{\beta+1}} d u=O(c) .
$$

The third term on the right in (2.77) works the same as the first term; $r^{\prime}(u) \theta^{\prime}(u)-$ $r(u) \theta^{\prime \prime}(u)$ is negative for all $c u>0$, and

$$
\int_{1}^{w} \frac{-3 i c e^{i\left(u^{2}+c u^{3}\right)}}{u(2+3 c u)^{2}(1+3 c u)^{\beta}} d u=O(c) .
$$

The fourth term works like the second term, except that the interval of integration must be cut at $c u=\left(2 \beta+9-\sqrt{4 \beta^{2}+20 \beta+1}\right) / 6(\beta+5)$ instead of $\frac{1}{3}(1-\sqrt{1-4 /(\beta+5)})$. The integral of the fourth term from $u=1$ to $w$ is still $O(c)$.

For the fifth term, we have $r(u)=u^{-2}\left[1-\cos \left(c u^{3}\right)\right]$ and $\theta(u)=u^{2}$, so

$$
r^{\prime}(u) \theta^{\prime}(u)-r(u) \theta^{\prime \prime}(u)=-6 u^{-2}\left[1-\cos \left(c u^{3}\right)\right]+6 c u \sin \left(c u^{3}\right) .
$$

This is positive if and only if

$$
1-\cos \left(c u^{3}\right)<c u^{3} \sin \left(c u^{3}\right)
$$

(multiply both sides of $(2.91)$ by $6 u^{-2}$ ), so it is sufficient to have $0<c u^{3}<1$. Therefore, by Lemma 2.2,

$$
\left|\int_{1}^{c^{-1 / 3}} \frac{i e^{i u^{2}}\left[1-\cos \left(c u^{3}\right)\right]}{u^{2}} d u\right|<\frac{2\left[1-\cos \left(c\left(c^{-1 / 3}\right)^{3}\right)\right]}{2 c^{-1 / 3}\left(c^{-1 / 3}\right)^{2}}=(1-\cos 1) c .
$$

For the integral from $c^{-1 / 3}$ to $w$, we rewrite $e^{i u^{2}}\left[1-\cos \left(c u^{3}\right)\right]$ as $e^{i u^{2}}-\frac{1}{2} e^{i\left(u^{2}+c u^{3}\right)}-$ $\frac{1}{2} e^{i\left(u^{2}-c u^{3}\right)}$. By Lemma 2.2, we have

$$
\begin{gathered}
\left|\int_{c^{-1 / 3}}^{w} \frac{i e^{i u^{2}}}{u^{2}} d u\right|<\frac{2}{2 c^{-1 / 3}\left(c^{-1 / 3}\right)^{2}}=c, \\
\left|\int_{c^{-1 / 3}}^{w} \frac{i e^{i\left(u^{2}+c u^{3}\right)}}{2 u^{2}} d u\right|<\frac{2}{2 c^{-1 / 3}\left(2+3 c^{2 / 3}\right)\left(c^{-1 / 3}\right)^{2}}<\frac{1}{2} c,
\end{gathered}
$$

and

$$
\left|\int_{c^{-1 / 3}}^{w} \frac{i e^{i\left(u^{2}-c u^{3}\right)}}{2 u^{2}} d u\right|<\frac{2}{2 c^{-1 / 3}\left(2-3 c^{2 / 3}\right)\left(c^{-1 / 3}\right)^{2}}=\frac{c}{2-3 c^{2 / 3}}=O(c) .
$$

Note that for (2.94), we have

$$
r^{\prime}(u) \theta^{\prime}(u)-r(u) \theta^{\prime \prime}(u)=-3 u^{-2}(1+2 c u),
$$

which is negative for all $u>0$, and for (2.95) we have

$$
r^{\prime}(u) \theta^{\prime}(u)-r(u) \theta^{\prime \prime}(u)=-3 u^{-2}(1-2 c u),
$$


which is negative for all $u<\frac{1}{2} c^{-1}$ (and therefore all $u<w$ ). Putting together (2.92), (2.93), (2.94), and (2.95), we have

$$
\int_{1}^{w} \frac{i e^{i u^{2}}\left[1-\cos \left(c u^{3}\right)\right]}{u^{2}} d u=O(c) .
$$

For the sixth term on the right of (2.77), we have

$$
\begin{gathered}
r^{\prime}(u) \theta^{\prime}(u)-r(u) \theta^{\prime \prime}(u) \\
=\frac{3(2+3 c u)(1+3 c u)\left[1-(1+2 c u)(1+3 c u)^{\beta}\right]+6(\beta+1) c u+9(\beta+2) c^{2} u^{2}}{u^{2}(2+3 c u)(1+3 c u)^{\beta+1}} .
\end{gathered}
$$

The denominator of the right-hand side of (2.97) is positive, and the numerator is equal to $f(c u)$, where

$$
f(v)=3(2+3 v)(1+3 v)\left[1-(1+2 v)(1+3 v)^{\beta}\right]+6(\beta+1) v+9(\beta+2) v^{2}
$$

Now $f(0)=0$, and

$$
\begin{aligned}
f^{\prime}(v)= & 27(1+2 v)\left[1-(1+2 v)(1+3 v)^{\beta}\right] \\
& -3(2+3 v)(1+3 v)^{\beta}[2+3 \beta+6(\beta+1) v]+6(\beta+1)+18(\beta+2) v .
\end{aligned}
$$

Therefore $f^{\prime}(0)=-12 \beta-6<0$, and there must exist a positive number $v_{0}$ (which depends on $\beta$, but not on $c$ ) such that $f(v)<0$ for $0<v<v_{0}$. The right-hand side of (2.97) is therefore negative for $0<u<c^{-1} v_{0}$, and it follows from Lemma 2.2 that

$$
\left|\int_{1}^{v_{0} c^{-1}} \frac{i e^{i\left(u^{2}+c u^{3}\right)}\left[(2+3 c u)(1+3 c u)^{\beta}-2\right]}{2 u^{2}(2+3 c u)(1+3 c u)^{\beta}} d u\right|<\frac{(2+3 c)(1+3 c)^{\beta}-2}{(2+3 c)^{2}(1+3 c)^{\beta}}=O(c) .
$$

We are free to choose $v_{0} \leq \frac{1}{3}-\frac{1}{9} \sqrt{3}$. If $v_{0}=\frac{1}{3}-\frac{1}{9} \sqrt{3}$, then $v_{0} c^{-1}=w$, and we are finished with the sixth term on the right of (2.77). Otherwise, $v_{0} c^{-1}<w$, and we need an upper bound on the integral from $v_{0} c^{-1}$ to $w$. But the integrand in (2.100) is $O\left(c^{2}\right)$ for $v_{0} c^{-1} \leq u \leq w$, because of the $u^{2}$ in the denominator, and $w-v_{0} c^{-1}=O\left(c^{-1}\right)$. Therefore the integral from $v_{0} c^{-1}$ to $w$ is also $O(c)$, and

$$
\int_{1}^{w} \frac{i e^{i\left(u^{2}+c u^{3}\right)}\left[(2+3 c u)(1+3 c u)^{\beta}-2\right]}{2 u^{2}(2+3 c u)(1+3 c u)^{\beta}} d u=O(c) .
$$

For the seventh term, we have

$$
r^{\prime}(u) \theta^{\prime}(u)-r(u) \theta^{\prime \prime}(u)
$$

$$
=\frac{3(2-3 c u)(1-3 c u)\left[(1-2 c u)(1-3 c u)^{\beta}-1\right]+6(\beta+1) c u-9(\beta+2) c^{2} u^{2}}{u^{2}(2-3 c u)(1-3 c u)^{\beta+1}}
$$

(note that $r(u)$ is the absolute value of the term, and $(2-3 c u)(1-3 c u)^{\beta}-2$ is negative). Again the denominator is positive, because $c u<\frac{1}{3}$, and the numerator equals $f(c u)$, where

$(2.103) f(v)=3(2-3 v)(1-3 v)\left[(1-2 v)(1-3 v)^{\beta}-1\right]+6(\beta+1) v-9(\beta+2) v^{2}$.

As before, $f(0)=0$ and

$$
\begin{aligned}
f^{\prime}(v)= & -27(1-2 v)\left[(1-2 v)(1-3 v)^{\beta}-1\right] \\
& -3(2-3 v)(1-3 v)^{\beta}[2+3 \beta-6(\beta+1) v]+6(\beta+1)-18(\beta+2) v,
\end{aligned}
$$


so $f^{\prime}(0)=-6-12 \beta<0$. We now proceed as with the sixth term, splitting the interval of integration at $v_{0} c^{-1}$, where $v_{0}$ is chosen so that $f(v)<0$ for $0<v<v_{0}$. We conclude that

$$
\int_{1}^{w} \frac{i e^{i\left(u^{2}-c u^{3}\right)}\left[(2-3 c u)(1-3 c u)^{\beta}-2\right]}{2 u^{2}(2-3 c u)(1-3 c u)^{\beta}} d u=O(c) .
$$

We have now shown that the integral from 1 to $w$ of each of the seven terms on the right of $(2.77)$ is $O(c)$. This establishes (2.81), and it follows that the sum of the integrals on the left-hand side of $(2.78)$ is $O(c)$. In order to prove $(2.69)$, we must evaluate the sum of the integrals from $u=0$ to 1 .

Now

$$
\frac{e^{i\left(u^{2}+c u^{3}\right)}}{(1+3 c u)^{\beta}}+\frac{e^{i\left(u^{2}-c u^{3}\right)}}{(1-3 c u)^{\beta}}-2 e^{i u^{2}}
$$

$$
=e^{i\left(u^{2}+c u^{3}\right)}\left[(1+3 c u)^{-\beta}-1\right]+e^{i\left(u^{2}-c u^{3}\right)}\left[(1-3 c u)^{-\beta}-1\right]+2 e^{i u^{2}}\left[\cos \left(c u^{3}\right)-1\right] .
$$

For $0 \leq u \leq 1$, the first two terms on the right above are $O(c)$, and the third term is $O\left(c^{2}\right)$. Hence the integrals from 0 to 1 are all $O(c)$, and so is the integral of the left-hand side of (2.106). This, along with (2.78), establishes (2.69), which implies (2.63).

Substituting (2.63) into (2.62), and using the definition of $c$ in (2.60), we get

$$
\begin{gathered}
\int_{\sqrt{2 \pi m / 9 x}}^{\infty} \frac{e^{i\left(x y^{3}-2 \pi m y\right)}}{y^{\beta}} d y \\
=(2 \pi m)^{-(2 \beta+1) / 4}(3 x)^{(2 \beta-1) / 4} e^{-\frac{2}{9} \sqrt{3} i(2 \pi m)^{3 / 2} x^{-1 / 2}} \int_{-\infty}^{\infty} e^{i u^{2}} d u \\
+O\left(x^{\beta / 2} m^{-(\beta+2) / 2}\right) .
\end{gathered}
$$

From (2.56), (2.57), and (2.107), we see that the sum of the integrals (2.54) and (2.55) is also equal to the right-hand side of (2.107), because (2.56) and (2.57) get absorbed by the error term $O\left(x^{\beta / 2} m^{-(\beta+2) / 2}\right)$ from $(2.107)$.

Finally, we add (2.50), (2.53), (2.54), and (2.55), noting that $-e^{i u}+e^{-i u}=$ $-2 i \sin u$, and sum the integrals from $m=1$ to $\infty$. Substituting back into (2.5), and then into (2.1), we get Theorem 2 , in the case $2<\beta<3$.

The case $\beta=3$ requires only a few modifications. The term, linear in $x$, that we got by summing (2.50) and (2.53) over $m$, disappears in this case, because $3-\beta=0$. It is replaced by the linear term $-\frac{1}{2} i x$, which arises from the fact that

$$
\psi(0)=\lim _{y \rightarrow 0} \frac{e^{i x y^{3}}-1}{y^{3}}=i x,
$$

instead of 0 , as in (2.3). We also have

$$
\lim _{z \rightarrow 0} g(z)=\frac{-x}{2 \pi m},
$$

instead of 0 , as in (2.9). It follows that

$$
\int_{0}^{z} \frac{\left(e^{i x y^{3}}-1\right) e^{2 \pi i m y}}{y^{3}} d y=\frac{-x}{2 \pi m}+\int_{0}^{z} g^{\prime}(y) d y-\frac{i\left(e^{i x z^{3}}-1\right) e^{2 \pi i m z}}{2 \pi m z^{3}}
$$


so the sum

$$
\sum_{m=1}^{\infty} \int_{0}^{z} \frac{\left(e^{i x y^{3}}-1\right) e^{2 \pi i m y}}{y^{3}} d y
$$

diverges. Fortunately, we have

$$
\lim _{z \rightarrow 0} \bar{g}(z)=\frac{x}{2 \pi m},
$$

where

$$
\bar{g}(z)=\int_{0}^{z} \frac{\left(e^{i x y^{3}}-1\right) e^{-2 \pi i m y}}{y^{\beta}} d y-\frac{i\left(e^{i x z^{3}}-1\right) e^{-2 \pi i m z}}{2 \pi m z^{\beta}},
$$

SO

$$
\int_{0}^{z} \frac{\left(e^{i x y^{3}}-1\right) e^{-2 \pi i m y}}{y^{\beta}} d y=\frac{x}{2 \pi m}+\int_{0}^{z} \bar{g}(y) d y+\frac{i\left(e^{i x z^{3}}-1\right) e^{-2 \pi i m z}}{2 \pi m z^{\beta}}
$$

and

$$
\sum_{m=1}^{\infty}\left[\int_{0}^{z} \frac{\left(e^{i x y^{3}}-1\right) e^{2 \pi i m y}}{y^{3}} d y+\int_{0}^{z} \frac{\left(e^{i x y^{3}}-1\right) e^{-2 \pi i m y}}{y^{3}} d y\right]
$$

does converge. The rest of the proof is exactly the same as for the case $2<\beta<3$.

The case $3<\beta<4$ is a bit trickier. If we define $\psi(y)$ as in (2.2), then $\lim _{y \rightarrow 0} \psi(y)=\infty$, so instead we let

$$
\psi(y)=\frac{e^{i x y^{3}}-1-i x y^{3}}{y^{\beta}},
$$

and take $\psi(0)$ to be $\lim _{y \rightarrow 0} \psi(y)=0$. But (2.116) introduces another difficulty. Because $\beta-3<1, \psi$ is not $L^{1}$, and we can longer assume Lemma 2.1. Instead, we proceed as follows.

For each real $\lambda, 0<\lambda<1$, let

$$
\varphi_{\lambda}(y)= \begin{cases}\frac{e^{i x \lambda^{3}}-1}{\lambda^{\beta}} & \text { for } 0 \leq y \leq \lambda, \\ \frac{e^{i x y^{3}}-1}{y^{\beta}} & \text { for } y \geq \lambda .\end{cases}
$$

Then $\varphi_{\lambda}$ is continuous and $L^{1}$ for each $\lambda$, so Lemma 2.1 holds. Thus

$$
\begin{array}{r}
\frac{1}{2} \frac{e^{i x \lambda^{3}}-1}{\lambda^{\beta}}+\sum_{n=1}^{\infty} \frac{e^{i x n^{3}}-1}{n^{\beta}}=\frac{e^{i x \lambda^{3}}-1}{\lambda^{\beta-1}}+\int_{\lambda}^{\infty} \frac{e^{i x y^{3}}-1}{y^{\beta}} d y \\
+\sum_{m=1}^{\infty} \frac{\left(e^{i x \lambda^{3}}-1\right) \sin 2 \pi m \lambda}{\pi m \lambda^{\beta}}+2 \sum_{m=1}^{\infty} \int_{\lambda}^{\infty} \frac{\left(e^{i x y^{3}}-1\right) \cos 2 \pi m y}{y^{\beta}} d y .
\end{array}
$$

Now fix a positive integer $k$, and for each real $\lambda, 0<\lambda<1$, let

$$
\tau_{\lambda}(y)= \begin{cases}\lambda^{3-\beta} & \text { if } 0 \leq y \leq \lambda, \\ y^{3-\beta} & \text { if } \lambda \leq y \leq k \\ k^{3-\beta}\left(1-d \frac{y-k}{\lambda}\right) & \text { if } k \leq y \leq k+\lambda, \\ 0 & \text { if } y \geq k+\lambda\end{cases}
$$


Then $\tau_{\lambda}$ is continuous and $L^{1}$ for each $\lambda$ (and each $k$ ), so Lemma 2.1 holds. Thus

$$
\frac{1}{2} \tau_{\lambda}(0)+\sum_{n=1}^{\infty} \tau_{\lambda}(n)=\int_{0}^{\infty} \tau_{\lambda}(y) d y+2 \sum_{m=1}^{\infty} \int_{0}^{\infty} \tau_{\lambda}(y) \cos 2 \pi m y d y
$$

But

$$
\int_{0}^{\infty} \tau_{\lambda}(y) d y=\lambda^{4-\beta}+\int_{\lambda}^{k} y^{3-\beta} d y+k^{3-\beta} \int_{k}^{k+\lambda}\left(1-\frac{y-k}{\lambda}\right) d y
$$

$$
=\lambda^{4-\beta}+\frac{k^{4-\beta}}{4-\beta}-\frac{\lambda^{4-\beta}}{4-\beta}+\lambda k^{3-\beta}-\frac{1}{2} \lambda k^{3-\beta}=\frac{3-\beta}{4-\beta} \lambda^{4-\beta}+\frac{k^{4-\beta}}{4-\beta}+\frac{1}{2} \lambda k^{3-\beta},
$$

and

$$
\begin{gathered}
=\lambda^{3-\beta} \int_{0}^{\lambda} \cos 2 \pi m y d y+\int_{\lambda}^{k} y^{3-\beta} \cos 2 \pi m y d y \\
+k^{3-\beta} \int_{k}^{k+\lambda}\left(1-\frac{y-k}{\lambda}\right) \cos 2 \pi m y d y \\
=\frac{\lambda^{3-\beta} \sin 2 \pi m \lambda}{2 \pi m}+\int_{\lambda}^{k} y^{3-\beta} \cos 2 \pi m y d y+k^{3-\beta} \int_{0}^{\lambda}\left(1-\frac{u}{\lambda}\right) \cos 2 \pi m(u+k) d u \\
=\frac{\lambda^{3-\beta} \sin 2 \pi m \lambda}{2 \pi m}+\int_{\lambda}^{k} y^{3-\beta} \cos 2 \pi m y d y+\frac{k^{3-\beta}(1-\cos 2 \pi m \lambda)}{\lambda(2 \pi m)^{2}} .
\end{gathered}
$$

Therefore

$$
\begin{gathered}
\frac{1}{2} \lambda^{3-\beta}+\sum_{n=1}^{k} n^{3-\beta}=\frac{3-\beta}{4-\beta} \lambda^{4-\beta}+\frac{k^{4-\beta}}{4-\beta}+\frac{1}{2} \lambda k^{3-\beta} \\
+2 \lambda^{4-\beta} \sum_{m=1}^{\infty} \frac{\sin 2 \pi m \lambda}{2 \pi m \lambda}+2 \sum_{m=1}^{\infty} \int_{\lambda}^{k} y^{3-\beta} \cos 2 \pi m y d y+2 k^{3-\beta} \lambda \sum_{m=1}^{\infty} \frac{1-\cos 2 \pi m \lambda}{(2 \pi m \lambda)^{2}},
\end{gathered}
$$

and

$$
+2 \lambda^{4-\beta} \sum_{m=1}^{\infty} \frac{\sin 2 \pi m \lambda}{2 \pi m \lambda}+2 \sum_{m=1}^{\infty} \int_{\lambda}^{k} y^{3-\beta} \cos 2 \pi m y d y+2 k^{3-\beta} \lambda \sum_{m=1}^{\infty} \frac{1-\cos 2 \pi m \lambda}{(2 \pi m \lambda)^{2}} .
$$

Taking the limit as $k \rightarrow \infty$, we get

$$
\begin{gathered}
\lim _{k \rightarrow \infty}\left(\sum_{n=1}^{k} n^{3-\beta}-\frac{k^{4-\beta}}{4-\beta}\right) \\
=-\frac{1}{2} \lambda^{3-\beta}+\frac{3-\beta}{4-\beta} \lambda^{4-\beta}+2 \lambda^{4-\beta} \sum_{m=1}^{\infty} \frac{\sin 2 \pi m \lambda}{2 \pi m \lambda}+2 \sum_{m=1}^{\infty} \int_{\lambda}^{k} y^{3-\beta} \cos 2 \pi m y d y .
\end{gathered}
$$


But (2.118) implies that

$$
\begin{gathered}
\lim _{k \rightarrow \infty}\left(\sum_{n=1}^{k} \frac{e^{i x n^{3}}-1}{n^{\beta}}-\int_{0}^{k} \frac{e^{i x y^{3}}-1}{y^{\beta}} d y\right)=-\frac{1}{2} \frac{e^{i x \lambda^{3}}-1}{\lambda^{\beta}}+\frac{e^{i x \lambda^{3}}-1}{\lambda^{\beta-1}} \\
-\int_{0}^{\lambda} \frac{e^{i x y^{3}}-1}{y^{\beta}} d y+\sum_{m=1}^{\infty} \frac{\left(e^{i x \lambda^{3}}-1\right) \sin 2 \pi m \lambda}{\pi m \lambda^{\beta}} \\
+2 \sum_{m=1}^{\infty} \int_{\lambda}^{\infty} \frac{\left(e^{i x y^{3}}-1\right) \cos 2 \pi m y}{y^{\beta}} d y .
\end{gathered}
$$

Multiplying equation (2.125) by $i x$, and subtracting it from equation (2.126), we get

$$
\begin{aligned}
& \lim _{k \rightarrow \infty}\left[\sum_{n=1}^{\infty} \psi(n)-\int_{0}^{k} \psi(y) d y\right]=-\frac{1}{2} \psi(\lambda)+\frac{e^{i x \lambda^{3}}-1}{\lambda^{\beta-1}}+\frac{\beta-3}{4-\beta} i x \lambda^{4-\beta} \\
& -\int_{0}^{\lambda} \frac{e^{i x y^{3}}-1}{y^{\beta}} d y+2 \psi(\lambda) \lambda \sum_{m=1}^{\infty} \frac{\sin 2 \pi m \lambda}{2 \pi m \lambda}+2 \sum_{m=1}^{\infty} \int_{\lambda}^{\infty} \psi(y) \cos 2 \pi m y d y
\end{aligned}
$$

where $\psi$ is defined in (2.116).

We now take the limit of both sides of (2.127) as $\lambda \rightarrow 0$. Note that the Taylor series of $\psi(y)$ is

$$
\psi(y)=\sum_{j=2}^{\infty} \frac{(i x)^{j} y^{3 j-\beta}}{j !},
$$

so that $\psi(\lambda)$ behaves like $\lambda^{6-\beta}$ as $\lambda \rightarrow 0$. Becuase $\beta<4$, the first four terms on the right-hand side of (2.127) tend to 0 as $\lambda \rightarrow 0$. The fifth term also tends to 0 , because

$$
\lim _{\lambda \rightarrow 0} \lambda \sum_{m=1}^{\infty} \frac{\sin 2 \pi m \lambda}{2 \pi m \lambda}=\frac{1}{2 \pi} \int_{0}^{\infty} \frac{\sin u}{u} d u .
$$

We want to show that the sixth term approaches

$$
2 \sum_{m=1}^{\infty} \int_{0}^{\infty} \psi(y) \cos 2 \pi m y d y
$$

and it suffices to show that

$$
\lim _{\lambda \rightarrow 0} \sum_{m=1}^{\infty} \int_{0}^{\lambda} \psi(y) \cos 2 \pi m y d y=0 .
$$

Integrating by parts twice, we get

$$
\begin{gathered}
\int_{0}^{\lambda} \psi(y) \cos 2 \pi m y d y \\
=\frac{\psi(\lambda) \sin 2 \pi m \lambda}{2 \pi m}+\frac{\psi^{\prime}(\lambda) \cos 2 \pi m \lambda}{(2 \pi m)^{2}}-\frac{1}{(2 \pi m)^{2}} \int_{0}^{\lambda} \psi^{\prime \prime}(y) \cos 2 \pi m y d y,
\end{gathered}
$$

since $\lim _{y \rightarrow 0} \psi(y)=0$. Indeed, differentiating (2.128) twice gives us

$$
\left|\psi^{\prime}(y)\right|=\left|\sum_{j=2}^{\infty} \frac{(i x)^{j}(3 j-\beta) y^{3 j-\beta-1}}{j !}\right|<\sum_{j=2}^{\infty} \frac{x^{2}(3 j) y^{5-\beta}}{j !}=3(e-1) x^{2} y^{5-\beta}
$$


and

$$
\begin{gathered}
\left|\psi^{\prime \prime}(y)\right|=\left|\sum_{j=2}^{\infty} \frac{(i x)^{j}(3 j-\beta)(3 j-\beta-1) y^{3 j-\beta-2}}{j !}\right| \\
<\sum_{j=2}^{\infty} \frac{9 j^{2}}{j !} x^{2} y^{4-\beta}<\sum_{j=2}^{\infty} \frac{18 j(j-1)}{j !} x^{2} y^{4-\beta}=18 e x^{2} y^{4-\beta}
\end{gathered}
$$

provided $x$ and $y$ are both less than 1 . Substituting the right-hand side of (2.132) for the integral in (2.131) gives us

$$
\begin{gathered}
\lim _{\lambda \rightarrow 0} \psi(\lambda) \sum_{m=1}^{\infty} \frac{\sin 2 \pi m \lambda}{2 \pi m}+\lim _{\lambda \rightarrow 0} \psi^{\prime}(\lambda) \sum_{m=1}^{\infty} \frac{\cos 2 \pi m \lambda}{(2 \pi m)^{2}} \\
-\lim _{\lambda \rightarrow 0} \sum_{m=1}^{\infty} \frac{1}{(2 \pi m)^{2}} \int_{0}^{\lambda} \psi^{\prime \prime}(y) \cos 2 \pi m y d y .
\end{gathered}
$$

The first term above is zero for the same reason that the fourth term on the righthand side of (2.127) tends to zero [see (2.129)]. The second term is bounded from above by

$$
\lim _{\lambda \rightarrow 0} \psi^{\prime}(\lambda) \sum_{m=1}^{\infty} \frac{1}{(2 \pi m)^{2}}=\lim _{\lambda \rightarrow 0} \frac{1}{24} \psi^{\prime}(\lambda),
$$

and is therefore zero by (2.133). The third term, by (2.134), is bounded by

$$
\lim _{\lambda \rightarrow 0} 18 e x^{2} \int_{0}^{\lambda} y^{4-\beta} d y \sum_{m=1}^{\infty} \frac{1}{(2 \pi m)^{2}}=\frac{3 e x^{2}}{4(5-\beta)} \lim _{\lambda \rightarrow 0} \lambda^{5-\beta},
$$

and is therefore also zero. Thus (2.131) holds, and (2.127) becomes

$$
\lim _{k \rightarrow \infty}\left[\sum_{n=1}^{k} \psi(n)-\int_{0}^{k} \psi(y) d y\right]=2 \sum_{m=1}^{\infty} \int_{0}^{\infty} \psi(y) \cos 2 \pi m y d y .
$$

In other words, the one-sided Poisson summation formula holds for $\psi$, in effect, even though $\psi$ is not $L^{1}$.

To complete the proof of Theorem 2 , in the case $3<\beta<4$, we note that $(2.1)$ and (2.6) still hold in this case, so that

$$
\begin{aligned}
& \sum_{n=1}^{\infty} \frac{e^{i x n^{3}}}{n^{\beta}} \\
& =\sum_{n=1}^{\infty} \frac{1}{n^{\beta}}+x^{(\beta-1) / 3} \int_{0}^{\infty} \frac{e^{i u^{3}}-1}{u^{\beta}} d u+i x \lim _{k \rightarrow \infty}\left(\sum_{n=1}^{k} n^{3-\beta}-\int_{0}^{k} y^{3-\beta} d y\right) \\
& \quad+\lim _{k \rightarrow \infty}\left(\sum_{n=1}^{k} \frac{e^{i x n^{3}}-1-i x n^{3}}{n^{\beta}}-\int_{0}^{k} \frac{e^{i x y^{3}}-1-i x y^{3}}{y^{\beta}} d y\right),
\end{aligned}
$$

where the final limit is equal to

$$
2 \sum_{m=1}^{\infty} \int_{0}^{\infty} \frac{\left(e^{i x y^{3}}-1-i x y^{3}\right) \cos 2 \pi m y}{y^{\beta}} d y
$$


by (2.138). Therefore

$$
\begin{gathered}
=\sum_{n=1}^{\infty} \frac{1}{n^{\beta}}+x^{(\beta-1) / 3} \int_{0}^{\infty} \frac{e^{i u^{3}}-1}{u^{\beta}} d u+i x \lim _{k \rightarrow \infty}\left(\sum_{n=1}^{k} n^{3-\beta}-\int_{0}^{k} y^{3-\beta} d y\right) \\
+\sum_{m=1}^{\infty} \int_{0}^{\infty} \frac{\left(e^{i x y^{3}}-1-i x y^{3}\right) e^{2 \pi i m y}}{y^{\beta}} d y+\sum_{m=1}^{\infty} \int_{0}^{\infty} \frac{\left(e^{i x y^{3}}-1-i x y^{3}\right) e^{-2 \pi i m y}}{y^{\beta}} d y .
\end{gathered}
$$

We now proceed as in the case $2<\beta<3$, but instead of (2.7), we let

$$
g(z)=\int_{0}^{z} \frac{\left(e^{i x y^{3}}-1-i x y^{3}\right) e^{2 \pi i m y}}{y^{\beta}} d y+\frac{i\left(e^{i x z^{3}}-1-i x z^{3}\right) e^{2 \pi i m z}}{2 \pi m z^{\beta}} .
$$

Then $\lim _{z \rightarrow 0} g(z)=0$ and

$$
g^{\prime}(z)=\frac{-3 x\left(e^{i x z^{3}}-1\right) e^{2 \pi i m z}}{2 \pi m z^{\beta-2}}-\frac{i \beta\left(e^{i x z^{3}}-1-i x z^{3}\right) e^{2 \pi i m z}}{2 \pi m z^{\beta+1}},
$$

SO

$$
\begin{gathered}
\int_{0}^{z} \frac{\left(e^{i x y^{3}}-1-i x y^{3}\right) e^{2 \pi i m y}}{y^{\beta}} d y \\
=\frac{-i\left(e^{i x z^{3}}-1-i x z^{3}\right) e^{2 \pi i m z}}{z^{\beta}}-\int_{0}^{z} \frac{3 x\left(e^{i x y^{3}}-1\right) e^{2 \pi i m y}}{2 \pi m y^{\beta-2}} d y \\
-\int_{0}^{z} \frac{i \beta\left(e^{i x y^{3}}-1-i x y^{3}\right) e^{2 \pi i m y}}{2 \pi m y^{\beta+1}} d y .
\end{gathered}
$$

Now the first term on the right-hand side of (2.144) tends to 0 as $z \rightarrow \infty$, so

$$
\begin{gathered}
\int_{0}^{\infty} \frac{\left(e^{i x y^{3}}-1-i x y^{3}\right) e^{2 \pi i m y}}{y^{\beta}} d y= \\
=\frac{-3 x}{2 \pi m} \int_{0}^{\infty} \frac{\left(e^{i x y^{3}}-1\right) e^{2 \pi i m y}}{y^{\beta-2}} d y-\frac{i \beta}{2 \pi m} \int_{0}^{\infty} \frac{\left(e^{i x y^{3}}-1-i x y^{3}\right) e^{2 \pi i m y}}{y^{\beta+1}} d y .
\end{gathered}
$$

We evaluate the integrals on the right-hand side of (2.145) just as we did in the case $2<\beta<3$. That is, we define $\tilde{g}(z)$ and $\hat{g}(z)$ as in (2.17) and (2.18), so that (2.19), (2.20), (2.21), (2.22), (2.23), and (2.24) hold for all $z$. Letting $z \rightarrow \infty$, so that the second terms on the right-hand sides of (2.23) and (2.24) tend to 0 , we get

$$
\begin{gathered}
\int_{0}^{\infty} \frac{\left(e^{i x y^{3}}-1\right) e^{2 \pi i m y}}{y^{\beta-2}} d y=\int_{0}^{\infty} \tilde{g}^{\prime}(y) d y \\
=\frac{-3 x}{2 \pi m} \int_{0}^{\infty} y^{4-\beta} e^{i\left(x y^{3}+2 \pi m y\right)} d y-\frac{i(\beta-2)}{2 \pi m} \int_{0}^{\infty} \frac{\left(e^{i x y^{3}}-1\right) e^{2 \pi i m y}}{y^{\beta-1}} d y
\end{gathered}
$$

and

$$
=\frac{-3 x}{2 \pi m} \int_{0}^{\infty} \frac{\left(e^{i x y^{3}}-1\right) e^{2 \pi i m y}}{y^{\beta-1}} d y-\frac{i(\beta+1)}{2 \pi m} \int_{0}^{\infty} \frac{\left(e^{i x y^{3}}-1-i x y^{3}\right) e^{2 \pi i m y}}{y^{\beta+2}} d y .
$$


Note that the second integral on the right-hand side of (2.146) is the same as the first integral on the right-hand side of (2.147). We evaluate this integral, as well as the second integral on the right side of (2.147), by splitting it at $y=x^{-1 / 3}$. The integral from 0 to $x^{-1 / 3}$ is estimated using Taylor series, and the integral from $x^{-1 / 3}$ to $\infty$ is handled by applying Lemma 2.2 , just as we did in the case $2<\beta<3$. The first integral on the right of $(2.146)$ is split at $y=\sqrt{2 \pi m(4-\beta) / 3 x(\beta-2)}$, and Lemma 2.2 is applied to each half. This differs slightly from the case $2<$ $\beta<3$, where we split the integral at $y=z=\sqrt{2 \pi m / 9 x}$, because if $\beta>\frac{7}{2}$ then $\sqrt{2 \pi m(4-\beta) / 3 x(\beta-2)}<\sqrt{2 \pi m / 9 x}$, and in order to apply Lemma 2.2, we must split the integral at the point where $r^{\prime}(y) \theta^{\prime}(y)-r(y) \theta^{\prime \prime}(y)$ changes sign. But since $3<\beta<4, \sqrt{2 \pi m(4-\beta) / 3 x(\beta-2)}$ is still on the order of $m^{1 / 2} x^{-1 / 2}$, so our estimates for these integrals are the same as they were in the case $2<\beta<3$. Furthermore, for $3<\beta<4$, we still have $\beta / 2<2,(\beta-2) / 2<(\beta-1) / 3$, and $\beta / 2<(\beta+2) / 3$ [see (2.37), (2.38), and (2.47)], so we still have

$$
\int_{0}^{\infty} \frac{\left(e^{i x y^{3}}-1-i x y^{3}\right) e^{2 \pi i m y}}{y^{\beta}} d y=O\left(x^{\beta / 2} m^{-(\beta+2) / 2}\right) .
$$

The next to last term in (2.141) is therefore $O\left(x^{\beta / 2}\right)$.

We now consider the last term in (2.141). Equations (2.145), (2.146), and (2.147) still hold if we replace the exponent $2 \pi i m y$ by $-2 \pi i m y$ throughout and multiply the right side of each equation by -1 . The initial parts of the integrals on the right side of (2.147), from $y=0$ to $x^{-1 / 3}$, can still be evaluated using Taylor series, and Lemma 2.2 implies that

$$
\frac{x}{m^{2}} \int_{x^{-1 / 3}}^{\infty} \frac{e^{-2 \pi i m y}}{y^{\beta-1}} d y O\left(x^{(\beta+2) / 3} m^{-3}\right)
$$

and

$$
\frac{1}{m^{2}} \int_{x^{-1 / 3}}^{\infty} \frac{e^{-2 \pi i m y}}{y^{\beta+2}} d y=O\left(x^{(\beta+2) / 3} m^{-3}\right)
$$

Therefore

$$
\begin{gathered}
\int_{0}^{\infty} \frac{\left(e^{i x y^{3}}-1-i x y^{3}\right) e^{-2 \pi i m y}}{y^{\beta}} d y=\frac{9 x^{2}}{4 \pi^{2} m^{2}} \int_{0}^{\infty} y^{4-\beta} e^{i\left(x y^{3}-2 \pi m y\right)} d y \\
+\frac{6 i(\beta-1) x}{4 \pi^{2} m^{2}} \int_{x^{-1 / 3}}^{\infty} \frac{e^{i\left(x y^{3}-2 \pi m y\right)}}{y^{\beta-1}} d y \\
-\frac{\beta(\beta+1)}{4 \pi^{2} m^{2}} \int_{x^{-1 / 3}}^{\infty} \frac{e^{i\left(x y^{3}-2 \pi m y\right)}}{y^{\beta+2}} d y+O\left(x^{\beta / 2} m^{-3}\right) .
\end{gathered}
$$

Each of the three integrals on the right side of (2.151) can be evaluated in the same way as the integral in (2.58). The only difference is that the lower limits are $0, x^{-1 / 3}$, and $x^{-1 / 3}$ respectively, instead of $z$, and $\beta$ is replaced by $\beta-4, \beta-1$, and $\beta+2$ respectively. Indeed, (2.62) still holds when $z$ and $\beta$ are replaced in this way, where $c$ is still defined as in (2.60), and $w$ is still on the order of $c^{-1}$. (Specifically, $w=\frac{1}{3} c^{-1}$ when the lower limit is 0 , and $\frac{1}{3} c^{-1}-c^{-1 / 3}$ when the lower limit is $x^{-1 / 3}$.) We must establish that (2.63) continues to hold as well.

In other words, we want to show that

$$
\int_{-w}^{\infty} \frac{e^{i\left(u^{2}+c u^{3}\right)}}{(1+3 c u)^{\gamma}} d u=\int_{-\infty}^{\infty} e^{i u^{2}} d u+O(c)
$$


where $w$ is on the order of $c^{-1}$, and $-1<\gamma<0,2<\gamma<3$, or $5<\gamma<6$ (that is, $\gamma=\beta-4, \beta-1$, or $\beta+2$ ). In fact, we have already done the case $2<\gamma<3$, because we proved (2.63) for $2<\beta<3$. The other cases require only a few minor changes in the proof.

First, note that as long as $\beta>-2$, we can use Lemma 2.2 to prove that the integral in (2.64) is $O\left(w^{-1}\right)=O(c)$. Nor is there any need to modify (2.86) and (2.87), because $1-4 /(\beta+5)>0$ as long as $\beta>-1$. Indeed, the only parts of the proof that must be changed are those which deal with the fourth, sixth, and seventh terms on the right side of (2.77).

For the fourth term, ignoring the constant $3 c$, and replacing $\beta$ with $\gamma$, we have

$$
r^{\prime}(u) \theta^{\prime}(u)-r(u) \theta^{\prime \prime}(u)=\frac{-9(\gamma+5) c^{2} u^{2}+3(2 \gamma+9) c u-4}{u(2-3 c u)^{2}(1-3 c u)^{\gamma+1}} .
$$

The denominator of (2.153) is positive for $0<c u<\frac{1}{3}$. The numerator starts out negative, when $c u=0$. If $-1 \leq \gamma \leq \sqrt{6}-\frac{2}{5}$, then the discriminant $9(2 \gamma+9)^{2}-$ $144(\gamma+5)$ is negative or zero, and the numerator of (2.153) is negative or zero for all $c u$. Otherwise, if $\gamma>\sqrt{6}-\frac{5}{2}$, then the discriminant is positive, and therefore the numerator is positive for

$$
\frac{2 \gamma+9-\sqrt{4 \gamma^{2}+20 \gamma+1}}{6(\gamma+5)}<c u<\frac{2 \gamma+9+\sqrt{4 \gamma^{2}+20 \gamma+1}}{6(\gamma+5)} .
$$

If (2.153) is negative for $0<c u<\frac{1}{3}$, then by Lemma 2.2, the integral of the fourth term from $u=1$ to $w$ is bounded in absolute value by $2 r(1) / \theta^{\prime}(1)=O(1)$, so we get $O(c)$ when we include the factor $3 c$. This conclusion does not change if $\gamma=\sqrt{6}-\frac{5}{2}$, so that $(2.153)$ is zero at $u=c^{-1}(2 \gamma+9) / 6(\gamma+5)$ and negative everywhere else.

Suppose, on the other hand, that $c u$ is positive in the range (2.154). If $\frac{1}{3}$, and hence $c w$, falls within this range, then we must split the integral at $u=$ $c^{-1}\left(2 \gamma+9-\sqrt{4 \gamma^{2}+20 \gamma=1}\right) / 6(\gamma+5)$ and apply Lemma 2.2 to each piece. We get a bound of $2 r(1) / \theta^{\prime}(1)$ for the first piece and $2 r(w) / \theta^{\prime}(w)$ for the second. If the entire interval in (2.154) is less than $\frac{1}{3}$, then we must split the integral at both $u_{1}=$ $c^{-1}\left(2 \gamma+9-\sqrt{\gamma^{2}+20 \gamma+1}\right) / 6(\gamma+5)$ and $u_{2}=c^{-1}\left(2 \gamma+9+\sqrt{\gamma^{2}+20 \gamma+1}\right) / 6(\gamma+5)$. The first piece is still bounded by $2 r(1) / \theta^{\prime}(1)$, and the second and third pieces are both bounded by $2 r\left(u_{2}\right) / \theta^{\prime}\left(u_{2}\right)$. But $u_{2}>c^{-1}\left[\frac{1}{3}-\frac{1}{6}\left(\frac{5}{2}-\sqrt{6}\right)^{-1}\right]$ for $\gamma>\sqrt{6}-\frac{5}{2}$, so $2 r\left(u_{2}\right) / \theta^{\prime}\left(u_{2}\right)$ is still $O(c)$. In other words, whether we evaluate the integral of the fourth term in one, two, or three pieces, the whole thing is always $O(c)$.

For the sixth term, we have

$$
r(u)=\frac{\left|(2+3 c u)(1+3 c u)^{\gamma}-2\right|}{2 u^{2}(2+3 c u)(1+3 c u)^{\gamma}} .
$$

Let

$$
h(v)=(2+3 v)(1+3 v)^{\gamma}-2 .
$$

Then $h(0)=0, h^{\prime}(0)=3(1+2 \gamma)$, and $h^{\prime \prime}(0)=18 \gamma^{2}$. Note that $h^{\prime}(0)$ is $>,=$, or $<0$ depending on whether $\gamma$ is $>,=$, or $<-\frac{1}{2}$, and $h^{\prime \prime}(0)>0$ if $\gamma=-\frac{1}{2}$. Therefore, for sufficiently small positive $v, h(v)$ is positive when $\gamma \geq-\frac{1}{2}$ and negative when 
$\gamma<-\frac{1}{2}$. Thus for $c u$ positive, but close to 0 , we have

$$
r(u)=\frac{(2+3 c u)(1+3 c u)^{\gamma}-2}{2 u^{2}(2+3 c u)(1+3 c u)^{\gamma}}
$$

if $\gamma \geq-\frac{1}{2}$, and

$$
r(u)=\frac{2-(2+3 c u)(1+3 c u)^{\gamma}}{2 u^{2}(2+3 c u)(1+3 c u)^{\gamma}}
$$

if $\gamma<-\frac{1}{2}$. That means equations (2.97), (2.98), and (2.99) hold, with $\gamma$ replacing $\beta$, if $\gamma \geq-\frac{1}{2}$; otherwise the right-hand side of each equation should be multiplied by -1 .

It follows that $f^{\prime}(0)=-12 \gamma-6$ if $\gamma>-\frac{1}{2}$ and $f^{\prime}(0)=12 \gamma+6$ if $\gamma<-\frac{1}{2}$. In either case, $f^{\prime}(0)<0$. If $\gamma=-\frac{1}{2}$, then $f^{\prime}(0)=0$, but $f^{\prime \prime}(0)=-54 \gamma^{2}-162 \gamma-72=-\frac{9}{2}$. So whatever the value of $\gamma$, there is always a positive number $v_{0}$ such that $f(v)<0$ for $0<v<v_{0}$. We can therefore apply Lemma 2.2 to the integral of the sixth term from $u=1$ to $v_{0} c^{-1}$, and conclude that this integral is $O(c)$.

We can use essentially the same argument for the seventh term. In this case we have

$$
r(u)=\frac{\left|(2-3 c u)(1-3 c u)^{\gamma}-2\right|}{2 u^{2}(2-3 c u)(1-3 c u)^{\gamma}}
$$

so we let

$$
h(v)=(2-3 v)(1-3 v)^{\gamma}-2 .
$$

We still have $h(0)=0$ and $h^{\prime \prime}(0)=18 \gamma^{2}$, but $h^{\prime}(0)=-3(1+2 \gamma)$, so for small positive $v, h(v)$ is positive when $\gamma \leq-\frac{1}{2}$ and negative when $\gamma>-\frac{1}{2}$. Thus equations (2.103) and (2.104) remain unchanged (except that $\gamma$ replaces $\beta$ ) when $\gamma>-\frac{1}{2}$, but the right sides of these equations are multiplied by -1 when $\gamma \leq-\frac{1}{2}$. As before, whether $\gamma$ is $>,=$, or $<-\frac{1}{2}$, either we have $f^{\prime}(0)<0$ or we have $f^{\prime}(0)=0$ and $f^{\prime \prime}(0)<0$, so we can still find $v_{0}>0$ such that $f(v)<0$ for $0<v<v_{0}$.

We conclude that (2.152) holds for all $\gamma>-1$. It follows from the analog of (2.62) that

$$
\begin{gathered}
\int_{z}^{\infty} \frac{e^{i\left(x y^{3}-2 \pi m y\right)}}{y^{\gamma}} d y \\
=(2 \pi m)^{-(2 \gamma+1) / 4}(3 x)^{(2 \gamma-1) / 4} e^{-\frac{2}{9} \sqrt{3} i(2 \pi m)^{3 / 2} x^{-1 / 2}} \int_{-\infty}^{\infty} e^{i u^{2}} d u \\
+O\left(m^{-(\gamma+2) / 2} x^{\gamma / 2}\right),
\end{gathered}
$$

where $3<\beta<4, \gamma=\beta-4, \beta-1$, or $\beta+2$, and $z=0$ or $x^{-1 / 3}$. Note that the error term is $O\left(\mathrm{~cm}^{-(2 \gamma+1) / 4} x^{(2 \gamma-1) / 4}\right)$, where $c$ is defined in (2.60).

We now apply (2.161) to each of the integrals on the right side of (2.151). For $\gamma=\beta-4$, we get

$$
\begin{gathered}
\frac{9 x^{2}}{4 \pi^{2} m^{2}} \int_{0}^{\infty} y^{4-\beta} e^{i\left(x y^{3}-2 \pi m y\right)} d y \\
=(2 \pi m)^{-(2 \beta+1) / 4}(3 x)^{(2 \beta-1) / 4} e^{-\frac{2}{9} \sqrt{3} i(2 \pi m)^{3 / 2} x^{-1 / 2}} \int_{-\infty}^{\infty} e^{i u^{2}} d u \\
+O\left(m^{-(\beta+2) / 2} x^{\beta / 2}\right),
\end{gathered}
$$


but the other two integrals in (2.151) are absorbed by the error term

$$
O\left(m^{-(\beta+2) / 2} x^{\beta / 2}\right) .
$$

Equations (2.141), (2.148), (2.151), and (2.162) suffice to establish Theorem 2 in the case $3<\beta<4$.

\section{BEHAVIOR NEAR THE RATIONAL POINTS}

We now prove Theorem 3 .

Proof. We start with the case $2<\beta \leq 3$. We have

$$
\begin{gathered}
F_{\beta}\left(\frac{p \pi}{q}+x\right)-F_{\beta}\left(\frac{p \pi}{q}\right)=\sum_{n=1}^{\infty} \frac{e^{i(p \pi / q+x) n^{3}}-e^{i p \pi n^{3} / q}}{n^{\beta}} \\
=\sum_{n=1}^{\infty}\left(\frac{e^{i x n^{3}}-1}{n^{\beta}}\right) e^{\pi i p n^{3} / q}=\sum_{k=1}^{2 q} e^{\pi i p k^{3} / q} \sum_{j=0}^{\infty} \frac{e^{i x(2 j q+k)^{3}}-1}{(2 j q+k)^{\beta}},
\end{gathered}
$$

letting $n=2 j q+k$. Now

$$
\sum_{j=0}^{\infty} \frac{e^{i x(2 j q+k)^{3}}-1}{(2 j q+k)^{\beta}}=\frac{1}{(2 q)^{\beta}} \sum_{j=0}^{\infty} \frac{e^{i\left(8 q^{3} x\right)(j+k / 2 q)^{3}}-1}{(j+k / 2 q)^{\beta}} .
$$

Letting

$$
\psi(y)=\frac{e^{i\left(8 q^{3} x\right)(y+k / 2 q)^{3}}-1}{(y+k / 2 q)^{\beta}}
$$

and applying Lemma 2.1, we get

$$
\begin{gathered}
\sum_{j=0}^{\infty} \frac{e^{i\left(8 q^{3} x\right)(j+k / 2 q)^{3}}-1}{(j+k / 2 q)^{\beta}}=\frac{e^{i\left(8 q^{3} x\right)(k / 2 q)^{3}}-1}{2(k / 2 q)^{\beta}}+\int_{0}^{\infty} \frac{e^{i\left(8 q^{3} x\right)(y+k / 2 q)^{3}}-1}{(y+k / 2 q)^{\beta}} d y \\
+2 \sum_{m=1}^{\infty} \int_{0}^{\infty} \frac{\left[e^{i\left(8 q^{3} x\right)(y+k / 2 q)^{3}}-1\right] \cos 2 \pi m y}{(y+k / 2 q)^{\beta}} d y
\end{gathered}
$$

We shall analyze each of the terms on the right side of (3.4) by expanding it as a Taylor series in $8 q^{3} x$ or $k^{3} x$. It will be necessary to assume that $x$ is bounded from above by some constant times $q^{-3}$, and for the sake of concreteness, will will assume that $x<\frac{1}{8} q^{-3}$, although any such bound will do. Since $k \leq 2 q$, this implies that $x<k^{-3}$.

The first two terms are easily disposed of. We have

$$
\frac{e^{i\left(8 q^{3} x\right)(k / 2 q)^{3}}-1}{2(k / 2 q)^{\beta}}=\frac{e^{i k^{3} x}-1}{2(k / 2 q)^{\beta}}=\frac{1}{2} i k^{3-\beta}(2 q)^{\beta} x+O\left(q^{6} x^{2}\right)
$$

by expanding $e^{i k^{3} x}$ as a Taylor series in $k^{3} x$ and using the fact that $k \leq 2 q$. Likewise,

$$
\begin{gathered}
\int_{0}^{\infty} \frac{e^{i\left(8 q^{3} x\right)(y+k / 2 q)^{3}}-1}{(y+k / 2 q)^{\beta}} d y=\int_{k / 2 q}^{\infty} \frac{e^{8 i q^{3} x y^{3}}-1}{y^{\beta}} d y \\
=\int_{0}^{\infty} \frac{e^{8 i q^{3} x y^{3}}-1}{y^{\beta}} d y-\int_{0}^{k / 2 q} \frac{e^{8 i q^{3} x y^{3}}-1}{y^{\beta}} d y
\end{gathered}
$$


where

$$
\int_{0}^{\infty} \frac{e^{8 i q^{3} x y^{3}}-1}{y^{\beta}} d y=(2 q)^{\beta-1} x^{(\beta-1) / 3} \int_{0}^{\infty} \frac{e^{i u^{3}}-1}{u^{\beta}} d u
$$

and

$$
\begin{gathered}
\int_{0}^{k / 2 q} \frac{e^{8 i q^{3} x y^{3}}-1}{y^{\beta}} d y=(2 q)^{\beta-1} x^{(\beta-1) / 3} \int_{0}^{k x^{1 / 3}} \frac{e^{i u^{3}}-1}{u^{\beta}} d u \\
=\frac{i(2 q)^{\beta-1} k^{4-\beta} x}{4-\beta}+O\left(q^{6} x^{2}\right) .
\end{gathered}
$$

The first equation in (3.8) is obtained by the change of variables $u=2 q x^{1 / 3} y$, and the second equation by expanding the Taylor series of the integral.

We now consider the third term on the right-hand side of (3.4). We have

$$
\begin{gathered}
\\
2 \int_{0}^{\infty} \frac{\left[e^{8 i q^{3} x(y+k / 2 q)^{3}}-1\right] \cos 2 \pi m y}{(y+k / 2 q)^{\beta}} d y \\
=2 \int_{k / 2 q}^{\infty} \frac{\left[e^{8 i q^{3} x y^{3}}-1\right] \cos 2 \pi m(y-k / 2 q)}{y^{\beta}} d y \\
=\int_{k / 2 q}^{\infty} \frac{\left[e^{8 i q^{3} x y^{3}}-1\right] e^{2 \pi i m(y-k / 2 q)}}{y^{\beta}} d y+\int_{k / 2 q}^{\infty} \frac{\left[e^{8 i q^{3} x y^{3}}-1\right] e^{-2 \pi i m(y-k / 2 q)}}{y^{\beta}} d y \\
=e^{-\pi i m k / q} \int_{k / 2 q}^{\infty} \frac{\left[e^{8 i q^{3} x y^{3}}-1\right] e^{2 \pi i m y}}{y^{\beta}} d y \\
+e^{\pi i m k / q} \int_{k / 2 q}^{\infty} \frac{\left[e^{8 i q^{3} x y^{3}}-1\right] e^{-2 \pi i m y}}{y^{\beta}} d y .
\end{gathered}
$$

But we have already dealt with the above integrals, in slightly disguised form, in the course of proving Theorem 2. Indeed, the last two integrals in (3.9) are the same as the last two integrals in (2.5), except that the former are evaluated from $k / 2 q$ to $\infty$, instead of 0 to $\infty$, and the $x$ in each of the latter integrals is replaced by $8 q^{3} x$.

Using the results we obtained while proving Theorem 2, specifically $(2.50),(2.53)$, (2.56), (2.57), and (2.107), and substituting $8 q^{3} x$ for $x$, we get

$$
\begin{gathered}
e^{-\pi i m k / q} \int_{0}^{\infty} \frac{\left[e^{8 i q^{3} x y^{3}}-1\right] e^{2 \pi i m y}}{y^{\beta}} d y+e^{\pi i m k / q} \int_{0}^{\infty} \frac{\left[e^{8 i q^{3} x y^{3}}-1\right] e^{-2 \pi i m y}}{y^{\beta}} d y \\
=\frac{-8 q^{3}(3-\beta) x}{(2 \pi m)^{4-\beta}}\left(e^{-\pi i m k / q} \int_{0}^{\infty} \frac{e^{i u}}{u^{\beta-2}} d u-e^{\pi i m k / q} \int_{0}^{\infty} \frac{e^{-i u}}{u^{\beta-2}} d u\right) \\
+e^{\pi i m k / q}(2 \pi m)^{-(2 \beta+1) / 4}\left(24 q^{3} x\right)^{(2 \beta-1) / 4} e^{-\frac{2}{9} \sqrt{3} i(2 \pi m)^{3 / 2}(2 q)^{-3 / 2} x^{-1 / 2}} \int_{-\infty}^{\infty} e^{i u^{2}} d u \\
+O\left(q^{3 \beta / 2} x^{\beta / 2} m^{-(\beta+2) / 2}\right) .
\end{gathered}
$$

We must subtract

$$
e^{-\pi i m k / q} \int_{0}^{k / 2 q} \frac{\left[e^{8 i q^{3} x y^{3}}-1\right] e^{2 \pi i m y}}{y^{\beta}} d y+e^{\pi i m k / q} \int_{0}^{k / 2 q} \frac{\left[e^{8 i q^{3} x y^{3}}-1\right] e^{-2 \pi i m y}}{y^{\beta}} d y
$$

from (3.10) to get (3.9). 
To estimate (3.11), we define $g(z)$ as in (2.7), but with $8 q^{3} x$ replacing $x$. From (2.10) and (2.11), again with $8 q^{3} x$ replacing $x$, we conclude that

$$
\begin{gathered}
\int_{0}^{z} \frac{\left[e^{8 i q^{3} x y^{3}}-1\right] e^{2 \pi i m y}}{y^{\beta}} d y \\
=\frac{-8(3-\beta) q^{3} x}{2 \pi m} \int_{0}^{z} \frac{e^{2 \pi i m y}}{y^{\beta-2}} d y-\frac{24 q^{3} x}{2 \pi m} \int_{0}^{z} \frac{\left(e^{8 i q^{3} x y^{3}}-1\right) e^{2 \pi i m y}}{y^{\beta-2}} d y \\
-\frac{i \beta}{2 \pi m} \int_{0}^{z} \frac{\left(e^{8 i q^{3} x y^{3}}-1-8 i q^{3} x y^{3}\right) e^{2 \pi i m y}}{y^{\beta+1}} d y-\frac{i\left(e^{8 i q^{3} x z^{3}}-1\right) e^{2 \pi i m z}}{2 \pi m z^{\beta}},
\end{gathered}
$$

where the change of variables $u=2 \pi m y$ yields

$$
\frac{-8(3-\beta) q^{3} x}{2 \pi m} \int_{0}^{z} \frac{e^{2 \pi i m y}}{y^{\beta-2}} d y=\frac{-8(3-\beta) q^{3} x}{(2 \pi m)^{4-\beta}} \int_{0}^{2 \pi m z} \frac{e^{i u}}{u^{\beta-2}} d y .
$$

If $\beta=3$, we must add $g(0)=-4 q^{3} x / \pi m$ to the right side of (3.12). We can put bounds on the second and third terms on the right side of (3.12) by expanding the Taylor series of the exponential functions in the integrals. We have

$$
e^{8 i q^{3} x y^{3}}-1=\sum_{\lambda=1}^{\infty} \frac{\left(8 i q^{3} x y^{3}\right)^{\lambda}}{\lambda !},
$$

SO

$$
\int_{0}^{z} \frac{\left(e^{8 i q^{3} x y^{3}}-1\right) e^{2 \pi i m y}}{y^{\beta-2}} d y=\sum_{\lambda=1}^{\infty} \frac{\left(8 i q^{3} x\right)^{\lambda}}{\lambda !} \int_{0}^{z} y^{3 \lambda-\beta+2} e^{2 \pi i m y} d y
$$

where

$$
\left|\int_{0}^{z} y^{3 \lambda-\beta+2} e^{2 \pi i m y} d y\right|<\frac{z^{3 \lambda-\beta+2}}{\pi m}
$$

by Lemma 2.2. Thus,

$$
\begin{aligned}
\left|\frac{24 q^{3} x}{2 \pi m} \int_{0}^{z} \frac{\left(e^{8 i q^{3} x y^{3}}-1\right) e^{2 \pi i m y}}{y^{\beta-2}} d y\right| & <\frac{24 q^{3} x}{2 \pi^{2} m^{2} z^{\beta-2}} \sum_{\lambda=1}^{\infty} \frac{\left(8 q^{3} x z^{3}\right)^{\lambda}}{\lambda !} \\
& =\frac{24 q^{3} x\left(e^{8 q^{3} x z^{3}}-1\right)}{2 \pi^{2} m^{2} z^{\beta-2}} .
\end{aligned}
$$

Likewise,

$$
e^{8 i q^{3} x y^{3}}-1-8 i q^{3} x y^{3}=\sum_{\lambda=2}^{\infty} \frac{\left(8 i q^{3} x y^{3}\right)^{\lambda}}{\lambda !},
$$

$\mathrm{SO}$

$$
\left|\frac{i \beta}{2 \pi m} \int_{0}^{z} \frac{\left(e^{8 i q^{3} x y^{3}}-1-8 i q^{3} x y^{3}\right) e^{2 \pi i m y}}{y^{\beta+1}} d y\right|<\frac{\beta\left(e^{8 q^{3} x z^{3}}-1-8 q^{3} x z^{3}\right)}{2 \pi^{2} m^{2} z^{\beta+1}} .
$$

We now set $z=k / 2 q$, so that $8 q^{3} x z^{3}=k^{3} x<1$. Since the functions $e^{u}-1$ and $e^{u}-1-u$ have strictly positive second and third derivatives, we have $e^{u}-1<(e-1) u$ and $e^{u}-1-u<(e-2) u^{2}$ for $0<u<1$, and in particular for $u=k^{3} x$. Therefore

$$
\begin{aligned}
\left|\frac{24 q^{3} x}{2 \pi m} \int_{0}^{k / 2 q} \frac{\left(e^{8 i q^{3} x y^{3}}-1\right) e^{2 \pi i m y}}{y^{\beta-2}} d y\right| & <\frac{3(e-1) k^{5-\beta}(2 q)^{\beta+1} x^{2}}{2 \pi^{2} m^{2}} \\
& \leq \frac{3(e-1)(2 q)^{6} x^{2}}{2 \pi^{2} m^{2}}
\end{aligned}
$$


and

$$
\left|\frac{i \beta}{2 \pi m} \int_{0}^{k / 2 q} \frac{\left(e^{8 i q^{3} x y^{3}}-1-8 i q^{3} x y^{3}\right) e^{2 \pi i m y}}{y^{\beta+1}} d y\right|<\frac{\beta(e-2)(2 q)^{6} x^{2}}{2 \pi^{2} m^{2}} .
$$

From (3.12), (3.13), (3.20), and (3.21), we conclude that

$$
\begin{gathered}
\int_{0}^{k / 2 q} \frac{\left[e^{8 i q^{3} x y^{3}}-1\right] e^{2 \pi i m y}}{y^{\beta}} d y \\
=\frac{-i\left(e^{i k^{3} x}-1\right) e^{\pi i m k / q}}{2 \pi m(k / 2 q)^{\beta}}-\frac{8(3-\beta) q^{3} x}{(2 \pi m)^{4-\beta}} \int_{0}^{\pi m k / q} \frac{e^{i u}}{u^{\beta-2}} d u+O\left(q^{6} x^{2} m^{-2}\right) .
\end{gathered}
$$

Again, we must add the term $-4 q^{3} x / \pi m$ when $\beta=3$. By a parallel line of reasoning, we find that

$$
\begin{gathered}
\int_{0}^{k / 2 q} \frac{\left[e^{8 i q^{3} x y^{3}}-1\right] e^{-2 \pi i m y}}{y^{\beta}} d y \\
=\frac{i\left(e^{i k^{3} x}-1\right) e^{-\pi i m k / q}}{2 \pi m(k / 2 q)^{\beta}}+\frac{8(3-\beta) q^{3} x}{(2 \pi m)^{4-\beta}} \int_{0}^{\pi m k / q} \frac{e^{-i u}}{u^{\beta-2}} d u+O\left(q^{6} x^{2} m^{-2}\right),
\end{gathered}
$$

plus $4 q^{3} x / \pi m$ when $\beta=3$. It follows that

$$
\begin{gathered}
e^{-\pi i m k / q} \int_{0}^{k / 2 q} \frac{\left[e^{8 i q^{3} x y^{3}}-1\right] e^{2 \pi i m y}}{y^{\beta}} d y+e^{\pi i m k / q} \int_{0}^{k / 2 q} \frac{\left[e^{8 i q^{3} x y^{3}}-1\right] e^{-2 \pi i m y}}{y^{\beta}} d y \\
=\frac{-8 q^{3}(3-\beta) x}{(2 \pi m)^{4-\beta}}\left(e^{-\pi i m k / q} \int_{0}^{\pi m k / q} \frac{e^{i u}}{u^{\beta-2}} d u-e^{\pi i m k / q} \int_{0}^{\pi m k / q} \frac{e^{-i u}}{u^{\beta-2}} d u\right) \\
+O\left(q^{6} x^{2} m^{-2}\right) .
\end{gathered}
$$

(Note that the first terms on the right sides of (3.22) and (3.23) cancel. In the case $\beta=3$, the terms $-4 q^{3} x / \pi m$ and $4 q^{3} x / \pi m$ also cancel.)

Finally, we subtract (3.24) from (3.10), use the identity

$$
\begin{gathered}
e^{-\pi i m k / q} \int_{\pi m k / q}^{\infty} \frac{e^{i u}}{u^{\beta-2}} d u-e^{\pi i m k / q} \int_{\pi m k / q}^{\infty} \frac{e^{-i u}}{u^{\beta-2}} d u \\
=-2 i \Re\left(i e^{-\pi i m k / q} \int_{\pi m k / q}^{\infty} \frac{e^{i u}}{u^{\beta-2}} d u\right),
\end{gathered}
$$

and sum over $m$. Note that when we sum over $m$, the error terms

$$
O\left(q^{3 \beta / 2} x^{\beta / 2} m^{-(\beta+2) / 2}\right) \text { and } O\left(q^{6} x^{2} m^{-2}\right)
$$

become

$$
O\left(q^{3 \beta / 2} x^{\beta / 2}\right) \text { and } O\left(q^{6} x^{2}\right)
$$

respectively, and the latter term is absorbed by the former. This establishes Theorem 3 in the case $2<\beta \leq 3$. 
For the case $3<\beta<4$, we start with (3.1) and (3.2), but follow with the observation that

$$
\sum_{j=0}^{\infty} \frac{e^{i\left(8 q^{3} x\right)(j+k / 2 q)^{3}}-1}{(j+k / 2 q)^{\beta}}
$$

$$
\begin{gathered}
\int_{0}^{\infty} \frac{e^{i\left(8 q^{3} x\right)(y+k / 2 q)^{3}}-1}{(y+k / 2 q)^{\beta}} d y+8 i q^{3} x \lim _{t \rightarrow \infty}\left[\sum_{j=0}^{t-1}\left(j+\frac{k}{2 q}\right)^{3-\beta}-\int_{0}^{t}\left(y+\frac{k}{2 q}\right)^{3-\beta} d y\right] \\
+\lim _{t \rightarrow \infty}\left[\sum_{j=0}^{t-1} \frac{e^{i\left(8 q^{3} x\right)(j+k / 2 q)^{3}}-1-i\left(8 q^{3} x\right)(j+k / 2 q)^{3}}{(j+k / 2 q)^{\beta}}\right. \\
\left.-\int_{0}^{t} \frac{e^{i\left(8 q^{3} x\right)(y+k / 2 q)^{3}}-1-i\left(8 q^{3} x\right)(y+k / 2 q)^{3}}{(y+k / 2 q)^{\beta}} d y\right] .
\end{gathered}
$$

We then let

$$
\psi(y)=\frac{e^{i\left(8 q^{3} x\right)(y+k / 2 q)^{3}}-1-i\left(8 q^{3} x\right)(y+k / 2 q)^{3}}{(y+k / 2 q)^{\beta}},
$$

and prove that

$$
\lim _{t \rightarrow \infty}\left[\sum_{n=0}^{t-1} \psi(n)-\int_{0}^{t} \psi(y) d y\right]=2 \sum_{m=1}^{\infty} \int_{0}^{\infty} \psi(y) \cos 2 \pi m y d y,
$$

just as we proved (2.138) in the course of proving Theorem 2. The rest of the proof is along the same lines as the case $2<\beta \leq 3$. In this case, however, because of how we defined $\psi(y)$, the right-hand side of (3.28) makes no contribution to the linear term, although the chirp term remains unchanged.

\section{BEHAVIOR NEAR THE IRRATIONAL POINTS}

Before proving Theorem 1, we will need several lemmas. The general idea is to show that $F_{\beta}(x)$ grows rapidly near suitable rational points $p \pi / q$. Lemma 4.1 shows that one can always find an interval near $p \pi / q$ where the sum of the $x^{(\beta-1) / 3}$ term and the linear term of $F_{\beta}(x)$ grows large, while the error term is still small. Lemma 4.2 shows that within this interval, one can always find points where the chirp term is small.

Lemma 4.1. Given $\beta(2<\beta<4)$ and given positive real numbers $c_{0}$ and $\varepsilon$, there exists a positive real number $c_{3}$ such that for all positive integers $p$ and $q$ there exist positive real numbers $c_{1}$ and $c_{2}$, bounded uniformly (over all $p$ and $q$ ) from above, such that if

$$
\left|\sum_{k=1}^{2 q} e^{\pi i p k^{3} / q}\right|>\varepsilon \sqrt{q}
$$

and

$$
c_{1} q^{-3(\beta+3) /(\beta+2)}<x<c_{2} q^{-3(\beta+3) /(\beta+2)},
$$

then both the real and imaginary parts of $\mathrm{H}_{1}^{+} x^{(\beta-1) / 3}+\mathrm{H}_{2}^{+}$ix (where $\mathrm{H}_{1}^{+}$and $\mathrm{H}_{2}^{+}$ are as defined in Theorem 3) are greater than $c_{3} q^{-\left(2 \beta^{2}+5 \beta-4\right) / 2(\beta+2)}$ in absolute value, while

$$
c_{0} q^{(\beta+2) / 2} x^{\beta / 2}<\frac{1}{3} c_{3} q^{-\left(2 \beta^{2}+5 \beta-4\right) / 2(\beta+2)} .
$$


Proof. First, note that the real and imaginary parts of $H_{1}^{+} x^{(\beta-1) / 3}$ are respectively

$$
\frac{x^{(\beta-1) / 3}}{2 q} \sum_{k=1}^{2 q} e^{\pi i p k^{3} / q} \int_{0}^{\infty} \frac{\cos u^{3}-1}{u^{\beta}} d u
$$

and

$$
\frac{x^{(\beta-1) / 3}}{2 q} \sum_{k=1}^{2 q} e^{\pi i p k^{3} / q} \int_{0}^{\infty} \frac{\sin u^{3}}{u^{\beta}} d u,
$$

because the sum in (4.1) and (4.2) is always real.

Now $\int_{0}^{\infty} u^{-\beta}\left(\cos u^{3}-1\right) d u$ and $\int_{0}^{\infty} u^{-\beta} \sin u^{3} d u$ are non-zero for all $\beta>2$. Indeed, $\int_{0}^{\infty} u^{-\beta}\left(\cos u^{3}-1\right) d u$ is strictly negative, because $u^{-\beta}\left(\cos u^{3}-1\right)<0$ for all $u$, except when $u^{3}$ is a multiple of $2 \pi$, in which case $u^{-\beta}\left(\cos u^{3}-1\right)=0$. On the other hand, letting $z=u^{3}$, we have

$$
\int_{0}^{\infty} \frac{\sin u^{3}}{u^{\beta}} d u=\int_{0}^{\infty} \frac{\sin z}{3 z^{(\beta+2) / 3}} d z
$$

and $\sin z / 3 z^{(\beta+2) / 3}$ is positive for $2 \mu \pi<z<(2 \mu+1) \pi$ and negative for $(2 \mu+1) \pi<$ $z<(2 \mu+2) \pi$, where $\mu$ is any non-negative integer. But

$$
\left|\frac{\sin (z+\pi)}{3(z+\pi)^{(\beta+2) / 3}}\right|=\left|\frac{\sin z}{3(z+\pi)^{(\beta+2) / 3}}\right|<\left|\frac{\sin z}{3 z^{(\beta+2) / 3}}\right|
$$

for all positive $z$. Thus the integral

$$
\int_{2 \mu \pi}^{2(\mu+1) \pi} \frac{\sin z}{3 z^{(\beta+2) / 3}} d z
$$

is strictly positive, and so is the right side (and hence the left side) of (4.3).

Let $K=-\Re\left(i H_{2}^{+}\right) / \Re\left(H_{1}^{+}\right)$. We have already established that the integral in (4.1) is non-zero. By hypothesis, the sum in (4.1) is also non-zero (it is $>\varepsilon \sqrt{q}$ in absolute value). Therefore $\Re\left(H_{1}^{+}\right)$is non-zero, and $K$ exists. $K$ is of course a function of $p, q$, and $\beta$.

We will show that, for suitable $c_{1}, c_{2}$, and $c_{3}$,

$$
\left|\frac{x^{(\beta-1) / 3}-K x}{2 q} \sum_{k=1}^{2 q} e^{\pi i p k^{3} / q} \int_{0}^{\infty} \frac{\cos u^{3}-1}{u^{\beta}} d u\right|<c_{3} q^{-\left(2 \beta^{2}+5 \beta-4\right) / 2(\beta+2)}
$$

and

$$
c_{0} q^{(\beta+2) / 2} x^{\beta / 2}<\frac{1}{3} c_{3} q^{-\left(2 \beta^{2}+5 \beta-4\right) / 2(\beta+2)}
$$

if

$$
c_{1} q^{-3(\beta+3) /(\beta+2)}<x<c_{2} q^{-3(\beta+3) /(\beta+2)} .
$$

By hypothesis, $2<\beta<4$, so

$$
\frac{3}{\beta-1}>1
$$

and

$$
\frac{4-\beta}{3}\left(\frac{\beta-1}{3}\right)^{(\beta-1) /(4-\beta)}>0 .
$$


Thus there is a unique positive real number $w$ satisfying

$$
w^{3 /(\beta-1)}=w+\frac{4-\beta}{3}\left(\frac{\beta-1}{3}\right)^{(\beta-1) /(4-\beta)} .
$$

In fact, we have $w>1$.

Let $x_{0}$ be any positive real number, and consider the function

$$
f(x)=x^{(\beta-1) / 3}-K x
$$

over the interval $0<x \leq x_{0}$. If

$$
K x_{0}^{(4-\beta) / 3} \leq \frac{\beta-1}{3},
$$

then $f(x)$ is monotonically increasing over the interval, and the maximum value occurs at $x=x_{0}$. Otherwise the maximum occurs at

$$
x=\left(\frac{\beta-1}{3 K}\right)^{3 /(4-\beta)} .
$$

However, if

$$
K x_{0}^{(4-\beta) / 3} \geq w^{(4-\beta) /(\beta-1)},
$$

then

$$
f\left(x_{0}\right) \leq-f\left(\left(\frac{\beta-1}{3 K}\right)^{3 /(4-\beta)}\right),
$$

so once again $|f(x)|$ attains its maximum at $x=x_{0}$. In the case of (4.13), the maximum is $x_{0}^{(\beta-1) / 3}-K x_{0}$, but

$$
K \leq \frac{1}{3}(\beta-1) x_{0}^{-(4-\beta) / 3},
$$

SO

$$
K x_{0} \leq \frac{1}{3}(\beta-1) x_{0}^{(\beta-1) / 3}
$$

and

$$
x_{0}^{(\beta-1) / 3}-K x_{0}>\frac{1}{3}(4-\beta) x_{0}^{(\beta-1) / 3} .
$$

If the maximum of $|f(x)|$ occurs at (4.14), then

$$
K<w^{(4-\beta) /(\beta-1)} x_{0}^{-(4-\beta) / 3},
$$

so the maximum is

$$
\begin{gathered}
x^{(\beta-1) / 3}-K x \\
=\left(\frac{\beta-1}{3 K}\right)^{(\beta-1) /(4-\beta)}-K\left(\frac{\beta-1}{3 K}\right)^{3 /(4-\beta)} \\
=\left(\frac{\beta-1}{3 K}\right)^{(\beta-1) /(4-\beta)}-\frac{\beta-1}{3}\left(\frac{\beta-1}{3 K}\right)^{(\beta-1) /(4-\beta)} \\
=\frac{4-\beta}{3}\left(\frac{\beta-1}{3 K}\right)^{(\beta-1) /(4-\beta)}>\frac{4-\beta}{3}\left(\frac{\beta-1}{3}\right)^{(\beta-1) /(4-\beta)} w^{-1} x_{0}^{(\beta-1) / 3} .
\end{gathered}
$$

In the case of (4.15), the maximum of $|f(x)|$ is

$$
K x_{0}-x_{0}^{(\beta-1) / 3} \geq\left(w^{(4-\beta) /(\beta-1)}-1\right) x_{0}^{(\beta-1) / 3},
$$


but (4.11) implies that

$$
w^{(4-\beta) /(\beta-1)}-1=\frac{4-\beta}{3}\left(\frac{\beta-1}{3}\right)^{(\beta-1) /(4-\beta)} w^{-1},
$$

so the lower bounds in (4.21) and (4.22) are identical. Furthermore, since $(\beta-1) /(4-\beta)>0,(\beta-1) / 3<1$, and $w^{-1}<1$, we have

$$
\frac{4-\beta}{3}\left(\frac{\beta-1}{3}\right)^{(\beta-1) /(4-\beta)} w^{-1}<\frac{4-\beta}{3},
$$

so the same lower bound on $|f(x)|$ also holds for (4.19). In other words, no matter what values we choose for $K$ and $x_{0}$, there always exists an $x$ in the interval $0<x \leq x_{0}$ such that

$$
\left|x^{(\beta-1) / 3}-K x\right|>\left(w^{(4-\beta) /(\beta-1)}-1\right) x_{0}^{(\beta-1) / 3} .
$$

But $\left|x^{(\beta-1) / 3}-K x\right|$ is a continuous function of $x$, so there must exist an open interval $x_{1}<x<x_{2}$, where $x_{1}>0$ and $x_{2} \leq x_{0}$, such that, for all $x$ in this interval,

$$
\left|x^{(\beta-1) / 3}-K x\right|>\frac{1}{2}\left(w^{(4-\beta) /(\beta-1)}-1\right) x_{0}^{(\beta-1) / 3} .
$$

Let

$$
\delta=\frac{1}{2}\left(w^{(4-\beta) /(\beta-1)}-1\right),
$$

and

$$
x_{0}=\left(\frac{1}{6} \delta \varepsilon c_{0}^{-1}\left|\int_{0}^{\infty} \frac{\cos u^{3}-1}{u^{\beta}} d u\right|\right)^{6 /(\beta+2)} q^{-3(\beta+3) /(\beta+2)} .
$$

Let

$$
c_{1}=x_{1} q^{3(\beta+3) /(\beta+2)}
$$

and

$$
c_{2}=x_{2} q^{3(\beta+3) /(\beta+2)} .
$$

Then, since $x_{1}<x_{2} \leq x_{0}$, we have

$$
c_{1}<c_{2} \leq\left(\frac{1}{6} \delta \varepsilon c_{0}^{-1}\left|\int_{0}^{\infty} \frac{\cos u^{3}-1}{u^{\beta}} d u\right|\right)^{6 /(\beta+2)},
$$

which establishes that $c_{1}$ and $c_{2}$ are bounded from above by a number independent of $p$ and $q$.

Let

$$
c_{3}=\left(3 c_{0}\right)^{-2(\beta-1) /(\beta+2)}\left(\frac{1}{2} \delta \varepsilon\left|\int_{0}^{\infty} \frac{\cos u^{3}-1}{u^{\beta}} d u\right|\right)^{3 \beta /(\beta+2)} .
$$

From (4.26), (4.27), and (4.28), we get, for $x_{1}<x<x_{2}$, that

$$
\left|x^{(\beta-1) / 3}-K x\right|>\delta\left(\frac{1}{6} \delta \varepsilon c_{0}^{-1}\left|\int_{0}^{\infty} \frac{\cos u^{3}-1}{u^{\beta}} d u\right|\right)^{2(\beta-1) /(\beta+2)} q^{-(\beta+3)(\beta-1) /(\beta+2)},
$$


and hence

$$
\begin{gathered}
\left|\frac{x^{(\beta-1) / 3}-K x}{2 q} \sum_{k=1}^{2 q} e^{\pi i p k^{3} / q} \int_{0}^{\infty} \frac{\cos u^{3}-1}{u^{\beta}} d u\right| \\
>\left|x^{(\beta-1) / 3}-K x\right| \frac{1}{2 q} \varepsilon \sqrt{q}\left|\int_{0}^{\infty} \frac{\cos u^{3}-1}{u^{\beta}} d u\right| \\
>\left(3 c_{0}\right)^{-2(\beta-1) /(\beta+2)}\left(\frac{1}{2} \delta \varepsilon\left|\int_{0}^{\infty} \frac{\cos u^{3}-1}{u^{\beta}} d u\right|\right)^{3 \beta /(\beta+2)} q^{-\left(2 \beta^{2}+5 \beta-4\right) /(2(\beta+2)} \\
=c_{3} q^{-\left(2 \beta^{2}+5 \beta-4\right) /(2(\beta+2)} .
\end{gathered}
$$

Likewise,

$$
x^{\beta / 2}<x_{0}^{\beta / 2}=\left(\frac{1}{6} \delta \varepsilon c_{0}^{-1}\left|\int_{0}^{\infty} \frac{\cos u^{3}-1}{u^{\beta}} d u\right|\right)^{3 \beta /(\beta+2)} q^{-3 \beta(\beta+3) / 2(\beta+2)},
$$

so

$$
\begin{gathered}
c_{0} q^{(\beta+2) / 2} x^{\beta / 2}<c_{0}\left(\frac{1}{6} \delta \varepsilon c_{0}^{-1}\left|\int_{0}^{\infty} \frac{\cos u^{3}-1}{u^{\beta}} d u\right|\right)^{3 \beta /(\beta+2)} q^{-\left(2 \beta^{2}+5 \beta-4\right) / 2(\beta+2)} \\
=\frac{1}{3} c_{3} q^{-\left(2 \beta^{2}+5 \beta-4\right) / 2(\beta+2)} .
\end{gathered}
$$

This establishes Lemma 4.1 for the real part. A similar argument works for the imaginary part, except that throughout the argument, $\int_{0}^{\infty} u^{-\beta}\left(\cos u^{3}-1\right) d u$ must be replaced by $\int_{0}^{\infty} u^{-\beta} \sin u^{3} d u$.

Lemma 4.2. Let $c_{1}, c_{2}$, and $c_{3}$ be any positive real numbers, with $c_{1}<c_{2}$. For every positive integer $p$, and every sufficiently large positive integer $q$, there exists a real number $x$, with $c_{1} q^{-3(\beta+3) /(\beta+2)}<x<c_{2} q^{-3(\beta+3) /(\beta+2)}$, such that the real part of $H_{3}^{+}(3 x)^{(2 \beta-1) / 4}$ (where $H_{3}^{+}$is defined as in Theorem 3) is less than $\frac{1}{3} c_{3} q^{-\left(2 \beta^{2}+5 \beta-4\right) / 2(\beta+2)}$ in absolute value. The same is true of the imaginary part, although not necessarily at the same $x$.

Proof. Let

$$
g(z)=\sum_{m=1}^{\infty}(2 \pi m)^{-(2 \beta+1) / 4} e^{-\frac{1}{18} \sqrt{6} i(2 \pi m)^{3 / 2} z} \sum_{k=1}^{2 q} e^{\pi i\left(p k^{3}+m k\right) / q} \int_{-\infty}^{\infty} e^{i u^{2}} d u,
$$

$$
G(z)=3 \sqrt{6} i \sum_{m=1}^{\infty}(2 \pi m)^{-(2 \beta+7) / 4} e^{-\frac{1}{18} \sqrt{6} i(2 \pi m)^{3 / 2} z} \sum_{k=1}^{2 q} e^{\pi i\left(p k^{3}+m k\right) / q} \int_{-\infty}^{\infty} e^{i u^{2}} d u
$$

and

$$
\Gamma(z)=-54 \sum_{m=1}^{\infty}(2 \pi m)^{-(2 \beta+13) / 4} e^{-\frac{1}{18} \sqrt{6} i(2 \pi m)^{3 / 2} z} \sum_{k=1}^{2 q} e^{\pi i\left(p k^{3}+m k\right) / q} \int_{-\infty}^{\infty} e^{i u^{2}} d u .
$$

Then for all real numbers $a$ and $b, 0<a<b$, we have

$$
\int_{a}^{b} g(z) d z=G(b)-G(a)
$$


and

$$
\int_{a}^{b} G(z) d z=\Gamma(b)-\Gamma(a) .
$$

Suppose for some $M>0$ we have

$$
|\Re[g(z)]|>M
$$

for all $z$ in the interval $a \leq z \leq b$. Since $g(z)$ is continuous, $\Re[g(z)]$ cannot change sign between $a$ and $b$. Therefore, for $a \leq w \leq b$, we have

$$
\left|\int_{a}^{w} \Re[g(z)] d z\right|>(w-a) M .
$$

But

$$
\int_{a}^{w} \Re[g(z)] d z=\Re\left[\int_{a}^{w} g(z) d z\right]=\Re[G(w)-G(a)]=\Re[G(w)]-\Re[G(a)]
$$

so

$$
|\Re[G(w)]-\Re[G(a)]|>(w-a) M
$$

for all $w$ between $a$ and $b$. Furthermore, $\Re[G(w)]-\Re[G(a)]$ has the same sign for all $w$ between $a$ and $b$.

If $\Re[G(a)]$ and $\Re[G(b)]$ have the same sign, then

$$
\left|\int_{a}^{b} \Re[G(w)] d w\right|>\frac{1}{2}(b-a)^{2} M .
$$

If, on the other hand, $\Re[G(w)]$ changes sign between $a$ and $b$, then, since it is monotonic, it can only change sign once. Say it changes sign at $v$, where $a<v<b$. Then

$$
\left|\int_{a}^{v} \Re[G(w)] d w\right|>\frac{1}{2}(v-a)^{2} M
$$

and

$$
\left|\int_{v}^{b} \Re[G(w)] d w\right|>\frac{1}{2}(b-v)^{2} M .
$$

But either $v-a \geq \frac{1}{2}(b-a)$ or $b-v \geq \frac{1}{2}(b-a)$, so the right-hand side of either (4.47) or (4.48) is greater than $\frac{1}{8}(b-a)^{2} M$. But the left-hand sides of (4.47) and (4.48) are respectively equal to $|\Re[\Gamma(v)]-\Re[\Gamma(a)]|$ and $|\Re[\Gamma(b)]-\Re[\Gamma(v)]|$. Therefore, at least one of the numbers $|\Re[\Gamma(a)]|,|\Re[\Gamma(v)]|$, and $|\Re[\Gamma(b)]|$ must be greater than $\frac{1}{16}(b-a)^{2} M$.

However, by (4.39), we have

$$
|\Gamma(z)| \leq 108 q \sum_{m=1}^{\infty}(2 \pi m)^{-(2 \beta+13) / 4}\left|\int_{-\infty}^{\infty} e^{i u^{2}} d u\right|
$$

for all real $z$, and the same bound applies to $|\Re[\Gamma(z)]|$. Therefore, if the righthand side of (4.49) should be less than or equal to $\frac{1}{16}(b-a)^{2} M$, we would have a contradiction, and (4.42) would have to be false for some $z$ in the interval $a \leq z \leq b$.

Let $c_{1}$ and $c_{2}$ be any real numbers, with $0<c_{1}<c_{2}$, and let

$$
a=c_{2}^{-1 / 2} q^{3 / 2(\beta+2)}
$$


and

$$
b=c_{1}^{-1 / 2} q^{3 / 2(\beta+2)},
$$

so that $0<a<b$. Let

$$
c_{4}=1728\left(c_{1}^{-1 / 2}-c_{2}^{-1 / 2}\right)^{-2} \sum_{m=1}^{\infty}(2 \pi m)^{-(2 \beta+13) / 4}\left|\int_{-\infty}^{\infty} e^{i u^{2}} d u\right| .
$$

Then the right-hand side of (4.49) is $\leq \frac{1}{16}(b-a)^{2} M$ whenever

$$
M \geq c_{4} q^{(\beta-1) /(\beta+2)} \text {. }
$$

It follows that

$$
|\Re[g(z)]| \leq c_{4} q^{(\beta-1) /(\beta+2)}
$$

for some $z$ in the interval $a \leq z \leq b$. The same argument shows us that

$$
|\Im[g(z)]| \leq c_{4} q^{(\beta-1) /(\beta+2)}
$$

for some $z$ in this interval, although the real and imaginary bounds do not necessarily occur simultaneously for the same $z$.

Now

$$
c_{1} q^{-3(\beta+3) /(\beta+2)}<x<c_{2} q^{-3(\beta+3) /(\beta+2)}
$$

if and only if

$$
c_{2}^{-1 / 2} q^{3 / 2(\beta+2)}<q^{-3 / 2} x^{-1 / 2}<c_{1}^{-1 / 2} q^{3 / 2(\beta+2)} .
$$

Therefore, there must exist $x$ satisfying (4.56) such that

$$
\left|\Re\left[g\left(q^{-3 / 2} x^{-1 / 2}\right)\right]\right|<c_{4} q^{(\beta-1) /(\beta+2)},
$$

and the same bound holds (generally at a different $x$ ) for $\Im\left[g\left(q^{-3 / 2} x^{-1 / 2}\right)\right]$. For such $x$ we have

$$
\begin{gathered}
\left|(2 q)^{(2 \beta-3) / 4}(3 x)^{(2 \beta-1) / 4} g\left(q^{-3 / 2} x^{-1 / 2}\right)\right| \\
<c_{5} q^{(2 \beta-3) / 4} x^{(2 \beta-1) / 4} q^{(\beta-1) /(\beta+2)}=c_{5} q^{\left(2 \beta^{2}+5 \beta-10\right) / 4(\beta+2)} x^{(2 \beta-1) / 4} \\
<c_{5} q^{\left(2 \beta^{2}+5 \beta-10\right) / 4(\beta+2)} c_{2}^{(2 \beta-1) / 4} q^{-3(2 \beta-1)(\beta+3) / 4(\beta+2)}=c_{6} q^{-\left(4 \beta^{2}+10 \beta+1\right) / 4(\beta+2)},
\end{gathered}
$$

where

$$
c_{5}=2^{(2 \beta-3) / 4} 3^{(2 \beta-1) / 4} c_{4}
$$

and

$$
c_{6}=c_{5} c_{2}^{(2 \beta-1) / 4} .
$$

Note that $c_{6}$ does not depend on $q$.

For $q$ sufficiently large, we have

$$
c_{6} q^{-\left(4 \beta^{2}+10 \beta+1\right) / 4(\beta+2)}<\frac{1}{3} c_{3} q^{-\left(4 \beta^{2}+10 \beta-8\right) / 4(\beta+2)}=\frac{1}{3} c_{3} q^{-\left(2 \beta^{2}+5 \beta-4\right) / 2(\beta+2)} .
$$

But

$$
(2 q)^{(2 \beta-3) / 4} g\left(q^{-3 / 2} x^{-1 / 2}\right)=H_{3}^{+} .
$$

It follows that for some $x$ satisfying (4.56) we have

$$
\left|\Re\left[H_{3}^{+}(3 x)^{(2 \beta-1) / 4}\right]\right|<\frac{1}{3} c_{3} q^{-\left(2 \beta^{2}+5 \beta-4\right) / 2(\beta+2)},
$$


and the same bound holds for the imaginary part at a possibly different $x$ satisfying (4.56).

Lemma 4.3. Let $q$ be a prime, with $q \equiv 1 \bmod 3$, let $s$ be an integer not divisible by $q$, and let $\chi$ be a cubic character on the integers $\bmod q$. Then

$$
\sum_{n=0}^{q-1} e^{2 \pi i s n^{3} / q}=2 \Re\left(\sum_{n=0}^{q-1} \chi(n) e^{\pi i s n / q}\right) .
$$

Proof. we have

$$
\sum_{n=1}^{q-1} \chi(n) e^{2 \pi i s n / q}=\sum_{\chi(n)=1} e^{2 \pi i s n / q}+\omega \sum_{\chi(n)=\omega} e^{2 \pi i s n / q}+\omega^{2} \sum_{\chi(n)=\omega^{2}} e^{2 \pi i s n / q},
$$

where $\omega=e^{2 \pi i / 3}$. Now

$$
\sum_{n=0}^{q-1} e^{2 \pi i s n^{3} / q}=1+3 \sum_{\chi(n)=1} e^{2 \pi i s n / q}
$$

and

$$
\sum_{\chi(n)=1} e^{2 \pi i s n / q}+\sum_{\chi(n)=\omega} e^{2 \pi i s n / q}+\sum_{\chi(n)=\omega^{2}} e^{2 \pi i s n / q}=\sum_{n=1}^{q-1} e^{2 \pi i s n / q}=-1,
$$

SO

$$
\sum_{\chi(n)=\omega} e^{2 \pi i s n / q}+\sum_{\chi(n)=\omega^{2}} e^{2 \pi i s n / q}=-1-\sum_{\chi(n)=1} e^{2 \pi i s n / q} .
$$

Since $-1=(-1)^{3}$, we have $\chi(-1)=1$, so

$$
\chi(-n)=\chi(n) \chi(-1)=\chi(n) .
$$

Therefore

$$
\sum_{\chi(n)=1} e^{2 \pi i s n / q}=\frac{1}{2} \sum_{\chi(n)=1}\left(e^{2 \pi i s n / q}+e^{-2 \pi i s n / q}\right)
$$

and

$$
\Im\left(\sum_{\chi(n)=1} e^{2 \pi i s n / q}\right)=0
$$

likewise

$$
\Im\left(\sum_{\chi(n)=\omega} e^{2 \pi i s n / q}\right)=0
$$

and

$$
\Im\left(\sum_{\chi(n)=\omega^{2}} e^{2 \pi i s n / q}\right)=0
$$

It follows from (4.65), (4.71), (4.72), and (4.73) that

$$
\Re\left(\sum_{n=1}^{q-1} \chi(n) e^{2 \pi i s n / q}\right)=\sum_{\chi(n)=1} e^{2 \pi i s n / q}-\frac{1}{2} \sum_{\chi(n)=\omega} e^{2 \pi i s n / q}-\frac{1}{2} \sum_{\chi(n)=\omega^{2}} e^{2 \pi i s n / q},
$$


but by (4.68), the right-hand side of (4.74) is equal to

$$
\frac{1}{2}+\frac{3}{2} \sum_{\chi(n)=1} e^{2 \pi i s n / q},
$$

and by (4.66), this is equal to

$$
\frac{1}{2} \sum_{n=0}^{q-1} e^{2 \pi i s n^{3} / q} .
$$

Therefore, twice (4.76) is equal to twice the left-hand side of (4.74). This, together with the fact that $\chi(0)=0$, establishes Lemma 4.3.

Corollary 4.3.1. If $p$ is even, and $q$ does not divide $p$, then

$$
\sum_{n=1}^{2 q} e^{\pi i p n^{3} / q}=2 \Re\left(\sum_{n=1}^{2 q} \chi(n) e^{\pi i p n / q}\right) .
$$

Proof. If $p=2 s$, then

$$
\sum_{n=1}^{2 q} e^{\pi i p n^{3} / q}=2 \sum_{n=0}^{q-1} e^{2 \pi i s n^{3} / q}
$$

and

$$
\sum_{n=1}^{2 q} \chi(n) e^{\pi i p n / q}=2 \sum_{n=0}^{q-1} \chi(n) e^{2 \pi i s n / q} .
$$

We also need the following results of Gauss, Heath-Brown and Patterson, and Erdös.

Theorem (Gauss [4] art. 358). Let $q$, s, and $\chi$ be as in Lemma 4.3. Then

$$
\left(\sum_{n=0}^{q-1} \chi(n) e^{2 \pi i s n / q}\right)^{3}=q \tau,
$$

where $\tau$ is a prime element of $\mathbf{Z}[\omega]$ with norm $q$, which is independent of s. Specifically,

where

$$
\tau=\frac{1}{2}(a+3 b \sqrt{3} i),
$$

$$
a^{2}+27 b^{2}=4 q
$$

(so that $|\tau|=\sqrt{q}$ ), $a \equiv 1 \bmod 3$, and the sign of $b$ depends only on the choice of the character $\chi$.

An immediate consequence of Gauss's theorem is that

$$
\left|\sum_{n=0}^{q-1} \chi(n) e^{2 \pi i s n / q}\right|=\sqrt{q},
$$

and hence, for even $p$,

$$
\left|\sum_{n=1}^{2 q} \chi(n) e^{\pi i p n / q}\right|=2 \sqrt{q}
$$


Another consequence is that

$$
\sum_{n=1}^{2 q} \chi(n) e^{\pi i p n / q}=\xi \sum_{n=1}^{2 q} \chi(n) e^{2 \pi i n / q},
$$

where $\xi=1, \omega$, or $\omega^{2}$, depending on $p$.

Theorem (Heath-Brown and Patterson [8]). Fix a cubic character $\chi$, and let

$$
\theta(q)=\arg \left(\sum_{n=1}^{q-1} \chi(n) e^{2 \pi i n / q}\right)
$$

range over all primes $q \equiv 1 \bmod 3$. Then $\theta(q)$ is uniformly distributed over the unit circle.

A consequence of the above theorem is the following lemma.

Lemma 4.4. Let $0<\varepsilon<2$ and let $S$ be the set of primes $q$ such that for all even integers $p$ which are not divisible by $q$, we have

$$
\left|\sum_{n=1}^{2 q} e^{\pi i p n^{3} / q}\right|>\varepsilon \sqrt{q}
$$

Then $S$ has positive asymptotic density among the set of all primes.

Proof. If $0<\varepsilon<2$, then

$$
0<\arcsin \frac{1}{4} \varepsilon<\frac{\pi}{6}
$$

and the set of $\theta$ such that

$$
|\theta-(2 \mu+1) \pi / 6|>\arcsin \frac{1}{4} \varepsilon
$$

for all $\mu \in \mathbf{Z}_{6}$ has positive measure on the unit circle. It follows from the theorem of Heath-Brown and Patterson that the set of primes $q \equiv 1 \bmod 3$ such that $\theta(q)$ satisfies (4.83) has positive density in the set of all primes $\equiv 1 \bmod 3$, and hence positive density in the set of all primes. For any such $q$, (4.81) implies that

$$
\left|\arg \left(\sum_{n=1}^{q-1} \chi(n) e^{\pi i p n / q}\right) \pm \frac{\pi}{2}\right|>\arcsin \frac{1}{4} \varepsilon
$$

for all even $p$ not divisible by $q$. Thus

$$
\left|\Re\left(\sum_{n=1}^{q-1} \chi(n) e^{\pi i p n / q}\right)\right|>\frac{1}{4} \varepsilon\left|\sum_{n=1}^{q-1} \chi(n) e^{\pi i p n / q}\right|=\frac{1}{2} \varepsilon \sqrt{q}
$$

by (4.80), and by Corollary 4.3.1

$$
\left|\sum_{n=1}^{2 q} e^{\pi i p n^{3} / q}\right|>\varepsilon \sqrt{q}
$$

$S$ therefore contains a set of positive density, and must have positive density itself. 
Theorem (Erdös [3]). Let $\delta>0$ and let $S$ be a set of positive integers such that the sum

$$
\sum_{q \in S} \varphi(q) / q^{2}
$$

diverges, where $\varphi$ is Euler's function. Then for almost all irrational numbers $\xi$, there exists an infinite number of rational numbers $p / q$ with $(p, q)=1$, such that $q \in S$ and

$$
\left|\xi-\frac{p}{q}\right|<\frac{\delta}{q^{2}}
$$

Corollary. Erdös's theorem remains true if we add the hypothesis that the elements of $S$ are all odd, and the conclusion that the integers $p$ are all even.

Proof. First we note that the theorem remains true if we replace $\delta$ and $\xi$ by $\frac{1}{2} \delta$ and $\frac{1}{2} \xi$ respectively, because if $\delta>0$, then so is $\frac{1}{2} \delta$, and because the set of all real numbers $\xi$ such that $\frac{1}{2} \xi$ belongs to a set of measure zero has measure zero. Then we multiply both sides of the inequality by 2 . The new inequality is the same as the original, except that $p$ has been replaced by $2 p$. Since $q$ is odd and $(p, q)=1$, we still have $(2 p, q)=1$.

We are now ready to prove Theorem 1.

Proof. If $q$ is prime, then $\varphi(q)=q-1$. It follows from the prime number theorem that for $0<\varepsilon<2$, the set $S$ in Lemma 4.4 always satisfies Erdös's theorem. If we exclude the case $q=2$, then the corollary of Erdös's theorem is also satisfied. Therefore, given $0<\varepsilon<2$ and $\delta>0$, there exist, for almost every irrational $\xi$, an infinite number of rationals $p / q$, with $p$ even and $(p, q)=1$, such that

$$
\left|\xi \pi-\frac{p \pi}{q}\right|<\frac{\delta \pi}{q^{2}}
$$

and

$$
\left|\sum_{n=1}^{2 q} e^{\pi i p n^{3} / q}\right|>\varepsilon \sqrt{q}
$$

We now fix $\xi$ and apply Lemma 4.1 for all such $p$ and $q$, letting $\varepsilon$ be as above, and letting $c_{0}$ be the constant from Theorem 3. We conclude that there exists a positive real number $c_{3}$ such that for each $p$ and $q$ satisfying (4.87) and (4.88) there is an interval

$$
c_{1} q^{-3(\beta+3) /(\beta+2)}<x<c_{2} q^{-3(\beta+3) /(\beta+2)},
$$

where $c_{2}$ is bounded from above uniformly over all such $p$ and $q$, such that, for all $x$ in this interval,

$$
\begin{aligned}
& \left|\Re\left(H_{1}^{+} x^{(\beta-1) / 3}+H_{2}^{+} i x\right)\right|>c_{3} q^{-\left(2 \beta^{2}+5 \beta-4\right) / 2(\beta+2)}, \\
& \left|\Im\left(H_{1}^{+} x^{(\beta-1) / 3}+H_{2}^{+} i x\right)\right|>c_{3} q^{-\left(2 \beta^{2}+5 \beta-4\right) / 2(\beta+2)},
\end{aligned}
$$

and

$$
\left|H_{4}^{+} x^{\beta / 2}\right|<\frac{1}{3} c_{3} q^{-\left(2 \beta^{2}+5 \beta-4\right) / 2(\beta+2)} .
$$


Moreover, by Lemma 4.2, if $q$ is sufficiently large, then for some $x$ satisfying (4.89), we have

$$
\left|\Re\left(H_{3}^{+} x^{(2 \beta-1) / 4}\right)\right|<\frac{1}{3} c_{3} q^{-\left(2 \beta^{2}+5 \beta-4\right) / 2(\beta+2)},
$$

and for some (possibly different) $x$, we have

$$
\left|\Im\left(H_{3}^{+} x^{(2 \beta-1) / 4}\right)\right|<\frac{1}{3} c_{3} q^{-\left(2 \beta^{2}+5 \beta-4\right) / 2(\beta+2)} .
$$

It follows from Theorem 3 that for an infinite number of $p$ and $q$ (that is, for all $p$ and $q$ satisfying (4.87) and (4.88), with a finite number of exceptions where $q$ is too small to satisfy Lemma 4.2 ), there exist numbers $x_{R}$ and $x_{I}$, both satisfying (4.89), such that

$$
\left|\Re\left[F_{\beta}\left(\frac{p \pi}{q}+x_{R}\right)-F_{\beta}\left(\frac{p \pi}{q}\right)\right]\right|>\frac{1}{3} c_{3} q^{-\left(2 \beta^{2}+5 \beta-4\right) / 2(\beta+2)}
$$

and

$$
\left|\Im\left[F_{\beta}\left(\frac{p \pi}{q}+x_{I}\right)-F_{\beta}\left(\frac{p \pi}{q}\right)\right]\right|>\frac{1}{3} c_{3} q^{-\left(2 \beta^{2}+5 \beta-4\right) / 2(\beta+2)} .
$$

Now suppose

$$
2<\beta \leq \frac{\sqrt{97}-1}{4}
$$

Then

$$
\left(2 \beta^{2}+5 \beta-4\right) / 2(\beta+2) \leq 2
$$

and

$$
\frac{1}{3} c_{3} q^{-\left(2 \beta^{2}+5 \beta-4\right) / 2(\beta+2)} \geq \frac{1}{3} c_{3} q^{-2} .
$$

For each $q>2 \delta$, there can be at most one $p$ satisfying (4.87), because $p$ must be the closest integer to $\xi q$. So we can think of $p, x_{R}$, and $x_{I}$ as functions of $q$. The domain of these functions consists of the infinite set of integers $q$ for which (4.95) holds. Let

$$
\begin{gathered}
x_{1}=p \pi / q, \\
x_{2}=p \pi / q+x_{R}, \\
x_{3}=\xi \pi, \\
y_{1}=\Re\left[F_{\beta}\left(x_{1}\right)\right], \\
y_{2}=\Re\left[F_{\beta}\left(x_{2}\right)\right],
\end{gathered}
$$

and

$$
y_{3}=\Re\left[F_{\beta}\left(x_{3}\right)\right] .
$$

We are to think of $x_{1}, x_{2}, y_{1}$, and $y_{2}$ as functions of $q$ as well. By (4.87) we have

$$
\lim _{q \rightarrow \infty}\left(x_{1}-x_{3}\right)=0 \text {, }
$$

and by (4.89), with $x=x_{R}$, we have

$$
\lim _{q \rightarrow \infty}\left(x_{1}-x_{2}\right)=0,
$$


so that

$$
\lim _{q \rightarrow \infty}\left(x_{2}-x_{3}\right)=0
$$

also. Now suppose $\Re\left(F_{\beta}\right)$ has a derivative of $D$ at $x=\xi \pi$. Then

$$
\lim _{q \rightarrow \infty} \frac{y_{3}-y_{1}}{x_{3}-x_{1}}=\lim _{q \rightarrow \infty} \frac{y_{3}-y_{2}}{x_{3}-x_{2}}=D .
$$

We will show that (4.109) leads to a contradiction.

First, we have

$$
\begin{aligned}
\lim _{q \rightarrow \infty} & \left(\frac{y_{3}-y_{1}}{x_{3}-x_{1}}-\frac{y_{3}-y_{2}}{x_{3}-x_{2}}\right) \\
\quad= & \lim _{q \rightarrow \infty}\left(\frac{y_{1} x_{2}-y_{2} x_{1}+y_{2} x_{3}-y_{3} x_{2}+y_{3} x_{1}-y_{1} x_{3}}{\left(x_{3}-x_{1}\right)\left(x_{3}-x_{2}\right)}\right)=0 .
\end{aligned}
$$

For $i=1,2$, and 3 , let $P_{i}=\left(x_{i}, y_{i}\right)$, let $b_{i}$ be the length of the side of triangle $P_{1} P_{2} P_{3}$ opposite $P_{i}$, let $h_{i}$ be the length of the perpendicular from $P_{i}$ to the opposite side, and let $\theta_{i}$ be the interior angle at $P_{i}$. Note that $b_{i}, h_{i}$, and $\theta_{i}$ are well defined even if $P_{1}, P_{2}$, and $P_{3}$ are collinear. (It is not possible for two of these points to coincide, because the $x_{i}$ are all different.) Now the absolute value of the numerator of the second limit in (4.110) is twice the area of triangle $P_{1} P_{2} P_{3}$. Thus

$$
\left|y_{1} x_{2}-y_{2} x_{1}+y_{2} x_{3}-y_{3} x_{2}+y_{3} x_{1}-y_{1} x_{3}\right|=b_{3} h_{3} .
$$

By (4.87), we have

$$
\left|x_{3}-x_{1}\right|<\delta \pi q^{-2}
$$

By (4.89), with $x=x_{R}$, we have

$$
\left|x_{2}-x_{1}\right|<c_{2} q^{-3(\beta+3) /(\beta+2)} .
$$

But $3(\beta+3) /(\beta+2)>2$, so

$$
\left|x_{2}-x_{1}\right|<\delta \pi q^{-2}
$$

for $q$ sufficiently large, and

$$
\left|x_{3}-x_{2}\right|<2 \delta \pi q^{-2} \text {. }
$$

That means the denominator of the second limit in (4.110) is less than $2 \delta^{2} \pi^{2} q^{-4}$ in absolute value, and

$$
\lim _{q \rightarrow \infty} q^{4} b_{3} h_{3}=0 .
$$

But

$$
b_{3}>\left|y_{2}-y_{1}\right|>\frac{1}{3} c_{3} q^{-2}
$$

by (4.95) and (4.99), so

$$
\lim _{q \rightarrow \infty} q^{2} h_{3}=0
$$

and

$$
\lim _{q \rightarrow \infty} \frac{h_{3}}{b_{3}}=0 .
$$

Now

$$
h_{3}=b_{2} \sin \theta_{1}=b_{1} \sin \theta_{2} \text {, }
$$


so (4.119) implies that

$$
\lim _{q \rightarrow \infty} \frac{b_{2}}{b_{3}} \sin \theta_{1}=\lim _{q \rightarrow \infty} \frac{b_{1}}{b_{3}} \sin \theta_{2}=0 .
$$

Since $\left|\sin \theta_{1}\right|$ and $\left|\sin \theta_{2}\right|$ are both $\leq 1$, we can multiply the first limit in (4.121) by $\sin \theta_{2}$ and the second limit by $\sin \theta_{1}$ without changing their zero values. Thus

$$
\lim _{q \rightarrow \infty} \frac{b_{2}}{b_{3}} \sin \theta_{1} \sin \theta_{2}=0
$$

and

$$
\lim _{q \rightarrow \infty} \frac{b_{1}}{b_{3}} \sin \theta_{1} \sin \theta_{2}=0 .
$$

Adding (4.122) and (4.123), we have

$$
\lim _{q \rightarrow \infty} \frac{b_{1}+b_{2}}{b_{3}} \sin \theta_{1} \sin \theta_{2}=0 .
$$

But

$$
\frac{b_{1}+b_{2}}{b_{3}} \geq 1
$$

by the triangle inequality, so

$$
\lim _{q \rightarrow \infty} \sin \theta_{1} \sin \theta_{2}=0 .
$$

On the other hand, (4.109) implies that

$$
\lim _{q \rightarrow \infty} \sin \theta_{3}=0 .
$$

But $\theta_{1}+\theta_{2}+\theta_{3}=\pi$, so

$$
\lim _{q \rightarrow \infty} \sin \left(\theta_{1}+\theta_{2}\right)=\lim _{q \rightarrow \infty}\left(\sin \theta_{1} \cos \theta_{2}+\sin \theta_{2} \cos \theta_{1}\right)=0 .
$$

Now $\left|\cos \theta_{1}\right|$ and $\left|\cos \theta_{2}\right|$ are also $\leq 1$, so we can multiply both sides of (4.128) by $\sin \theta_{1} \cos \theta_{2}$ and both sides of (4.126) by $\cos \theta_{1} \cos \theta_{2}$, to get

$$
\lim _{q \rightarrow \infty}\left(\sin ^{2} \theta_{1} \cos ^{2} \theta_{2}+\sin \theta_{1} \sin \theta_{2} \cos \theta_{1} \cos \theta_{2}\right)=0
$$

and

$$
\lim _{q \rightarrow \infty} \sin \theta_{1} \sin \theta_{2} \cos \theta_{1} \cos \theta_{2}=0 .
$$

Subtracting (4.130) from (4.129) yields

$$
\lim _{q \rightarrow \infty} \sin ^{2} \theta_{1} \cos ^{2} \theta_{2}=0,
$$

and taking the square root of both sides gives us

$$
\lim _{q \rightarrow \infty} \sin \theta_{1} \cos \theta_{2}=0 .
$$

Adding $\sin \theta_{2}$ times (4.126) to $\cos \theta_{2}$ times (4.132), we get

$$
\lim _{q \rightarrow \infty} \sin \theta_{1}\left(\sin ^{2} \theta_{2}+\cos ^{2} \theta_{2}\right)=\lim _{q \rightarrow \infty} \sin \theta_{1}=0 .
$$


Recall that $\theta_{1}$ is the angle between segments $P_{1} P_{2}$ and $P_{1} P_{3}$. Thus (4.133) implies that in the limit as $q \rightarrow \infty$, both segments tend to the same slope. Therefore (4.109) implies that

$$
\lim _{q \rightarrow \infty} \frac{y_{2}-y_{1}}{x_{2}-x_{1}}=D
$$

But by (4.95) and (4.113), we have

$$
\left|\frac{y_{2}-y_{1}}{x_{2}-x_{1}}\right|>\frac{\frac{1}{3} c_{3} q^{-\left(2 \beta^{2}+5 \beta-4\right) / 2(\beta+2)}}{c_{2} q^{-3(\beta+3) /(\beta+2)}}=\frac{c_{3}}{3 c_{2}} q^{\left(22+\beta-2 \beta^{2}\right) / 2(\beta+2)} \text {. }
$$

Since $\beta \leq(\sqrt{97}-1) / 4$, we have

$$
\frac{2 \beta^{2}+5 \beta-4}{2(\beta+2)} \leq 2<\frac{3(\beta+3)}{\beta+2},
$$

so $\left(22+\beta-2 \beta^{2}\right) / 2(\beta+2)>0$ and

$$
\lim _{q \rightarrow \infty} \frac{c_{3}}{3 c_{2}} q^{\left(22+\beta-2 \beta^{2}\right) / 2(\beta+2)}=+\infty .
$$

Thus $D$ does not exist, and $\Re\left[F_{\beta}(x)\right]$ has no derivative at $x=\xi \pi$. A parallel argument proves that $\Im\left[F_{\beta}(x)\right]$ has no derivative at $x=\xi \pi$.

\section{ACKNOWLEDGMENTS}

I thank Paul Bateman, József Beck, Roger Heath-Brown, Martin Karel, S.J. Patterson, and Eli Stein for bringing some of the references to my attention, and for their other helpful advice.

\section{REFERENCES}

1. P.L. Butzer and E.L. Stark, "Riemann's example" of a continuous nondifferentiable function in the light of two letters (1865) of Cristoffel to Prym, Bull. Soc. Math. Belg. Sér. A, 38 (1986) 45-73. MR 88d:01007

2. J.J. Duistermaat, Self-similarity of "Riemann's nondifferentiable function", Nieuw Arch. Wisk. (4), 9 (1991) 303-337. MR 93h:26009

3. P. Erdös, On the distribution of the convergents of almost all real numbers, J. Number Theory, 2 (1970) 425-441. MR 42:5941

4. C.F. Gauss, Disquisitiones Arithmeticae, Lipsiae, 1801.

5. J. Gerver, The differentiability of the Riemann function at certain rational multiples of $\pi$, Amer. J. Math. 92 (1970) 33-55. MR 42:434

6. J. Gerver, More on the differentiability of the Riemann function, Amer. J. Math. 93 (1971) 33-41. MR 43:2169

7. G.H. Hardy, Weierstrass's non-differentiable function, Trans. Amer. Math. Soc. 17 (1916) 301-325.

8. G.H. Hardy and J.E. Littlewood, A new solution of Waring's problem, Quarterly J. Math. 48 (1920) 272-293.

9. D.R. Heath-Brown and S.J. Patterson, The distribution of Kummer sums at prime arguments, J. Reine Angew. Math. 310 (1979) 111-130. MR 81e:10033

10. M. Holschneider et P. Tchamitchian, Pointwise analysis of Riemann's nondifferentiable function, Inventiones Math. 105 (1991) 157-175. MR 93g:26010

11. S. Itatsu, Differentiability of Riemann's function, Proc. Japan Acad. Ser. A, 57 (1981) 492495. MR 83e:42006

12. S. Jaffard, The spectrum of singularities of Riemann's function, Rev. Mat. Iberoamericana, 12 (1996) 441-460. MR 97g:26006

13. S. Jaffard and Y. Meyer, Wavelet methods for pointwise regularity and local oscillations of functions, Mem. Amer. Math. Soc. 123 (1996), no. 587. MR 97d:42005 
14. W. Luther, The differentiability of Fourier gap series and "Riemann's example" of a continuous, nondifferentiable function, J. Approximation Theory, 48 (1986) 303-321. MR 88d:42018

15. E. Mohr, Wo ist die Riemannsche funktion nicht differenzierbar? Ann. Mat. Pura Appl. 123 (1980) 93-104. MR 81i:26005

16. E. Neuenschwander, Riemann's example of a continuous nondifferentiable function, Math. Intelligencer, 1 (1978) 40-44. MR 58:15962a

17. H. Queffelec, Dérivabilité de certaines sommes de séries de Fourier lacunaires, C.R. Acad. Sci. Paris, 273 (1971) 291-293. MR 44:4159

Department of Mathematics, Rutgers University, Camden, New Jersey 08102

E-mail address: gerver@camden.rutgers.edu 